\title{
Partition function of free conformal higher spin theory
}

\author{
Matteo Beccaria, ${ }^{a}$ Xavier Bekaert ${ }^{b}$ and Arkady A. Tseytlin ${ }^{c, 1}$ \\ ${ }^{a}$ Dipartimento di Matematica e Fisica Ennio De Giorgi, \\ Università del Salento and INFN, Via Arnesano, 73100 Lecce, Italy \\ ${ }^{b}$ Laboratoire de Mathématiques et Physique Théorique, \\ Unité Mixte de Recherche 7350 du CNRS, Fédération de Recherche 2964 Denis Poisson, \\ Université François Rabelais, Parc de Grandmont, 37200 Tours, France \\ ${ }^{c}$ The Blackett Laboratory, Imperial College, \\ London $S W 7$ 2AZ, U.K. \\ E-mail: matteo.beccaria@le.infn.it, \\ xavier.bekaert@lmpt.univ-tours.fr, tseytlin@imperial.ac.uk
}

AbSTRACT: We compute the canonical partition function $\mathcal{Z}$ of non-interacting conformal higher spin (CHS) theory viewed as a collection of free spin $s$ CFT's in $\mathbb{R}^{d}$. We discuss in detail the 4-dimensional case (where $s=1$ is the standard Maxwell vector, $s=2$ is the Weyl graviton, etc.), but also present a generalization for all even dimensions $d$. $\mathcal{Z}$ may be found by counting the numbers of conformal operators and their descendants (modulo gauge identities and equations of motion) weighted by scaling dimensions. This conformal operator counting method requires a careful analysis of the structure of characters of relevant (conserved current, shadow field and conformal Killing tensor) representations of the conformal algebra $\mathfrak{s o}(d, 2)$. There is also a close relation to massless higher spin partition functions with alternative boundary conditions in $\operatorname{AdS}_{d+1}$. The same partition function $\mathcal{Z}$ may also be computed from the CHS path integral on a curved $S^{1} \times S^{d-1}$ background. This allows us to determine a simple factorized form of the CHS kinetic operator on this conformally flat background. Summing the individual conformal spin contributions $\mathcal{Z}_{s}$ over all spins we obtain the total partition function of the CHS theory. We also find the corresponding Casimir energy on the sphere and show that it vanishes if one uses the same regularization prescription that implies the cancellation of the total conformal anomaly $a$-coefficient. This happens to be true in all even dimensions $d \geq 2$.

KEYwords: Higher Spin Symmetry, AdS-CFT Correspondence

ArXiv ePrint: 1406.3542

\footnotetext{
${ }^{1}$ Also at: Lebedev Institute, Moscow.
} 


\section{Contents}

1 Introduction and summary 2

2 Free CFT partition function: scalar and vector examples $\quad 7$

2.1 Operator counting method in $\mathbb{R}^{d} \quad 8$

2.2 Partition function on $S^{1} \times S^{d-1} \quad 9$

3 Conformal spin 2 in $d=4 \quad 10$

3.1 Quadratic fluctuation operator in conformally flat background 11

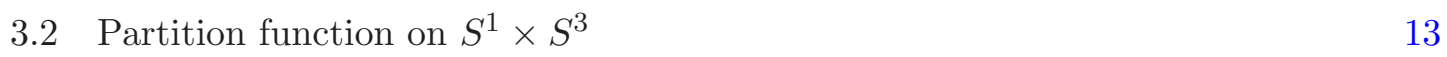

3.3 Partition function from conformal operator counting in $\mathbb{R}^{4} \quad 14$

4 Partition function of general conformal higher spin field in $d=4 \quad 15$

5 Conformal spin $s$ partition function from $\mathrm{CFT}_{d} / \mathrm{AdS}_{d+1}$ perspective $\quad 17$

$\begin{array}{ll}5.1 d=4 \text { case } & 17\end{array}$

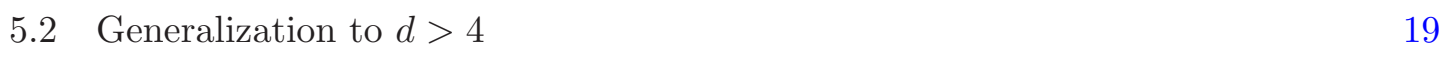

5.3 Partition functions from characters of conformal algebra representations 21

6 Summing over spins: total CHS partion function and vanishing of Casimir energy 23

$\begin{array}{lll}7 & \text { Concluding remarks } & 26\end{array}$

$\begin{array}{ll}\text { A Spectrum of spin } s \text { laplacian on } S^{d} & 27\end{array}$

$\begin{array}{ll}\text { B Expansions of curvature-squared invariants } & 27\end{array}$

C Factorized form of Weyl graviton operator on an Einstein background 28

$\begin{array}{ll}\text { D On tensor zero modes on } S^{3} & 28\end{array}$

$\begin{array}{lll}\mathrm{E} \text { Characters of } \mathrm{SO}(d) \text { representations } & 29\end{array}$

\begin{tabular}{ll} 
F Characters of relevant $\mathfrak{s o}(d, 2)$ & representations \\
\hline
\end{tabular}

F.1 Characters of generalized Verma $\mathfrak{s o}(d, 2)$-modules $\quad 30$

F.2 Odd dimension $d \geqslant 3 \quad 31$

F.3 Even dimension $d \geqslant 4 \quad 34$ 


\section{Introduction and summary}

Conformal higher spin (CHS) theories are generalizations of $d=4$ Maxwell $(s=1)$ and Weyl $(s=2)$ theories that describe pure spin $s$ states off shell, i.e. have maximal gauge symmetry consistent with locality [1] (see also [2-4]). The free CHS action in flat 4dimensional space may be written as

$$
S_{s}=\int d^{4} x \phi_{s} P_{s} \partial^{2 s} \phi_{s}=\int d^{4} x(-1)^{s} C_{s} C_{s}
$$

where $\phi_{s}=\left(\phi_{\mu_{1} \ldots \mu_{s}}\right) \equiv \phi_{\mu(s)}$ is a totally symmetric tensor and $P_{s}=\left(P_{\nu_{1} \ldots \nu_{s}}^{\mu_{1} \ldots \mu_{s}}\right) \equiv P_{\nu(s)}^{\mu(s)}$ is the transverse projector which is traceless and symmetric within $\mu$ and $\nu$ groups of indices. This action is thus invariant under a combination of differential (analog of reparametrizations) and algebraic (analog of Weyl) gauge transformations: $\delta \phi_{s}=\partial \xi_{s-1}+g_{2} \eta_{s-2}$ (here $g_{2}$ is flat euclidean metric and $\xi$ and $\eta$ are parameter tensors $) . C_{s} \equiv C_{\mu(s), \nu(s)}=\left(C_{\mu_{1} \ldots \mu_{s}, \nu_{1} \ldots \nu_{s}}\right)$ is the generalized Weyl tensor, i.e. the gauge-invariant field strength that can be written as $C_{\mu(s), \nu(s)}=\mathcal{P}_{\mu(s), \nu(s)}^{\lambda(s), \rho(s)} \partial_{\lambda(s)}^{s} \phi_{\rho(s)}$. Here $\mathcal{P}_{s}$ is the projector ${ }^{1}$ that makes $C_{\mu(s), \nu(s)}$ totally symmetric and traceless in each group of indices $\mu(s)$ and $\nu(s)$ and also antisymmetric between them, so that $C_{\mu(s), \nu(s)}$ corresponds to the $(s, s)$ representation of $\mathrm{SO}(4)$ described by the rectangular two-row Young tableau. ${ }^{2}$ It is often convenient to write the components of $C_{s}$ as $C_{\mu_{1} \nu_{1} \mu_{2} \nu_{2} \ldots \mu_{s} \nu_{s}}$ with antisymmetry in each pair of $\mu_{i}$ and $\nu_{i}$ and total symmetry in $\mu$ 's and $\nu$ 's so that $C_{1}=\left(F_{\mu \nu}\right)$ is the Maxwell tensor, $C_{2}=\left(C_{\mu_{1} \nu_{1} \mu_{2} \nu_{2}}\right)$ is the linearized Weyl tensor, etc. (see $[5,6]$ ).

The analog of $d=4$ CHS action (1.1) in any even dimension $d$ is

$$
S_{s}=\int d^{d} x \phi_{s} P_{s} \partial^{2 s+d-4} \phi_{s}=(-1)^{s} \int d^{d} x C_{s} \partial^{d-4} C_{s}
$$

so that $\phi_{s}$ and $C_{s}$ have $d$-independent $\mathrm{SO}(d, 2)$ scaling dimensions

$$
\Delta\left(\phi_{s}\right)=2-s, \quad \Delta\left(C_{s}\right)=2 .
$$

The action (1.1), (1.2) formally defines a free higher-spin non-unitary CFT in $d$ dimensions. While in this paper we will discuss only free CHS theory which is a sum of individual free spin $s$ theories we shall emphasize the existence of its interacting generalization in the final section.

Our aim here will be to compute the associated one-particle or canonical partition function $\mathcal{Z}(q)=\sum_{n} \mathrm{~d}_{n} q^{\Delta_{n}}$ that counts the numbers of corresponding gauge-invariant conformal primaries and their descendants weighted with scaling dimensions like in the familiar $d=4$ free standard scalar and spin 1 cases in $[7,8] .^{3}$

\footnotetext{
${ }^{1}$ Note the identity $P_{\nu(s)}^{\rho(s)} \partial^{2 s}=\mathcal{P}_{\mu(s), \nu(s)}^{\lambda(s), \rho(s)} \partial_{\lambda(s)}^{s}\left(\partial^{s}\right)^{\mu(s)}$ leading to the second form of the action in (1.1) [2].

${ }^{2}$ In this paper we always use Young labels $\ell=\left(\ell_{1}, \ell_{2}, \ldots, \ell_{r}\right)$ to denote representation of $\mathrm{SO}(2 r): \ell_{i}$ are numbers of boxes in rows of the corresponding Young tableau.

${ }^{3}$ Note the the $s=0$ field which is the member of the CHS family is non-dynamical in $d=4$ and is the same as the standard 2-derivative conformal scalar field only in $d=6$.
} 
One should also find the same $\mathcal{Z}_{s}(q)$ from the standard finite temperature one-loop partition function $Z_{s}$ on $S^{1} \times S^{d-1}$ background (with euclidean time circle of length $\beta$ ) which may be interpreted as multi-particle or grand canonical partition function given by

$$
\ln Z_{s}=\sum_{m=1}^{\infty} \frac{1}{m} \mathcal{Z}_{s}\left(q^{m}\right), \quad q=e^{-\beta} .
$$

To compute $Z_{s}$ then requires the knowledge of the kinetic CHS operator on curved $S^{1} \times$ $S^{d-1}$ background.

The form of the covariant kinetic CHS operator $\mathcal{O}_{s}=D^{2 s+d-4}+\ldots$ on a curved background is not known in general ${ }^{4}$ but it should have a particularly simple structure on a conformally flat space. Indeed, it was found recently that on a conformally flat background which is also an Einstein space, like $S^{d}$ or $\mathrm{AdS}_{d}$, the operator $\mathcal{O}_{s}$ factorizes into a product of 2-nd derivative partially massless and massive spin $s$ operators [9-12].

Below we will determine the form of $\mathcal{O}_{s}$ on the conformally flat but non-Einstein $d=4$ background $S^{1} \times S^{3}$ by first (i) finding it explicitly in the familiar $s=1$ and $s=2$ cases, then (ii) conjecturing a natural generalization to the $s>2$ case, and finally (iii) checking the consistency of the resulting partition function $\mathcal{Z}_{s}$ with the one found by direct count of conformal CHS operators in $\mathbb{R}^{4}$ that can be justified by representation-theoretic methods. We will also find the expression for $\mathcal{Z}_{s}$ for all even dimensions $d>4$.

The study of this partition function is also of interest in the context of remarkable relations between conformal higher spin theory in $d$ dimensions, singlet sector of free scalar CFT in $\mathbb{R}^{d}$ and dual massless higher spin theory in $\operatorname{AdS}_{d+1}$. A free massless complex scalar theory $S=-\int d^{d} x \Phi_{r}^{*} \partial^{2} \Phi_{r} \quad(r=1, \ldots, N)$ has a tower of (on-shell) conserved symmetric traceless higher spin currents $J_{s} \sim P_{s} \Phi_{r}^{*} \partial^{s} \Phi_{r}, \partial J_{s}=0$ which are conformal fields of dimension $\Delta\left(J_{s}\right)=s+d-2 \equiv \Delta_{+}$. Adding these currents to the action with the source or shadow fields $\phi_{s}(x)$ one observes that this $\phi_{s}$ has the same dimension $2-s \equiv \Delta_{-}=d-\Delta_{+}$ and effectively the same algebraic and gauge (due to properties of $J_{s}$ ) symmetries as the CHS field in (1.3). Integrating out the free fields $\Phi_{r}$ in the path integral then gives an effective action for $\phi_{s}$ the leading local (logarithmically divergent) part of which is, at quadratic level, the same as the classical CHS action in (1.1), (1.2) [3, 13]. From the AdS/CFT perspective this induced action should be found upon the substitution of the solution of the Dirichlet problem (with $\phi_{s}$ as the boundary data) into the classical action of a massless spin $s$ field in $\mathrm{AdS}_{5}$.

In addition to this classical relation, there is also a one-loop one $[9,14]$

$$
\left.\frac{Z_{-s}}{Z_{+s}}\right|_{M^{d}}=\left.Z_{s}\right|_{M^{d}}
$$

Here $Z_{s}$ is the 1-loop CHS partition function on a conformally flat space $M^{d}$. $Z_{+s}$ is the free scalar CFT partition function in the spin $s$ current part of the singlet sec-

\footnotetext{
${ }^{4}$ This operator is expected to be reparametrization and Weyl invariant and consistent with CHS gauge symmetries for any background metric solving Bach equations of Weyl gravity theory.
} 
tor (the total singlet sector partition function is $\prod_{s} Z_{+s}$ ) and $Z_{-s}$ is its spin $s$ shadow operator counterpart. ${ }^{5}$

By the AdS/CFT rules, $Z_{ \pm s}$ should have the equivalent interpretation as the one-loop partition function $Z_{s}^{( \pm)}$of the massless higher spin $s$ field $\varphi_{s}$ in $\operatorname{AdS}_{d+1}$ with boundary $M^{d}$ computed with the standard $\varphi_{s} \sim z^{\Delta_{+}-s}$ or the alternative $\varphi_{s} \sim z^{\Delta_{-}-s}$ boundary conditions (see [14] and references there). Thus, we should also have the following relation between the massless higher spin partition functions in the bulk $\operatorname{AdS}_{d+1}$ and the CHS partition function at the conformally flat boundary $M^{d}$

$$
\left.\frac{Z_{s}^{(-)}}{Z_{s}^{(+)}}\right|_{A d S_{d+1}}=\left.Z_{s}\right|_{M^{d}} .
$$

These relations (1.5), (1.6) were verified explicitly $[9,10,14,15]$ in the case of $M^{d}$ being the sphere $S^{d}$ for even $d=4$ and $d=6$ where $\log Z_{s}$ is essentially determined by the conformal anomaly $a$-coefficient.

Below we will demonstrate the validity of these relations also in the case of $M^{d}=$ $S^{1} \times S^{d-1}$. In this case eqs. (1.5) or (1.6) may be written as a relation between the corresponding one-particle partition functions as functions of $q=e^{-\beta}$ (cf. (1.4))

$$
\mathcal{Z}_{-s}(q)-\mathcal{Z}_{+s}(q)=\mathcal{Z}_{s}(q), \quad \mathcal{Z}_{ \pm s}(q)=\mathcal{Z}_{s}^{( \pm)}(q)
$$

The expression for $\mathcal{Z}_{+s}$ is straightforward to find using the conformal operator counting method in $\mathbb{R}^{d}[7,8]$ : it should compute the operators represented by the components of the traceless symmetric spin $s$ current operator $J_{s}=\left(J_{\mu_{1} \ldots \mu_{s}}\right)$ of dimension $\Delta_{+}$and all of its conformal descendants (derivatives) modulo the conservation condition $\partial^{\mu_{1}} J_{\mu_{1} \ldots \mu_{s}}=0$ ( rank $s-1$ tensor of dimension $\Delta_{+}^{\prime}=\Delta_{+}+1$ ) and all of its derivatives. This immediately leads to

$$
\begin{gathered}
\mathcal{Z}_{+s}=\frac{\mathrm{n}_{s} q^{\Delta_{+}}-\mathrm{n}_{s-1} q^{\Delta_{+}^{\prime}}}{(1-q)^{d}}, \\
\Delta_{+}=s+d-2, \quad \Delta_{+}^{\prime}=s+d-1, \quad \mathrm{n}_{s}=(2 s+d-2) \frac{(s+d-3) !}{(d-2) ! s !},
\end{gathered}
$$

where $\mathrm{n}_{s}$ is the number of components of totally symmetric traceless rank $s$ tensor in $d$ dimensions. Explicitly, in $d=4$ this gives

$$
d=4: \quad \mathcal{Z}_{+s}=\frac{(s+1)^{2} q^{s+2}-s^{2} q^{s+3}}{(1-q)^{4}},
$$

\footnotetext{
${ }^{5}$ This relation can be motivated [14] by considering the double-trace $J_{s} J_{s}$ deformation of the free large $N$ scalar theory under which the scaling dimension of only one (spin $s$ ) operator is changed, i.e. the l.h.s. of (1.5) is $Z_{\mathrm{UV}} / Z_{\mathrm{IR}}$, i.e. the ratio of the UV and IR large $N$ fixed point CFT partition functions (this argument can be made precise in $d=3$ [14]). In even $d$ case that we are considering here $Z_{s}$ is given by the determinant of the local CHS kinetic operator while in odd $d$ the corresponding kinetic operator is the full nonlocal kernel $\left\langle J_{s} J_{s}\right\rangle$ appearing in the induced theory.
} 
eq. (1.8) is indeed the same as the massless spin $s$ field partition function $\mathcal{Z}_{s}^{(+)}$in thermal $\mathrm{AdS}_{d+1}$ with $S^{1} \times S^{d-1}$ boundary [16-18], with the negative (current conservation subtraction) term here corresponding to the ghost contribution. ${ }^{6}$

A natural guess for the expression for $\mathcal{Z}_{-s}$ that should be counting the shadow spin $s$ operators (modulo gauge degeneracy) is just to replace the dimensions $\Delta_{+}$and $\Delta_{+}^{\prime}$ in (1.8) by their shadow $(\Delta \rightarrow d-\Delta)$ values, i.e.

$$
\widetilde{\mathcal{Z}}_{-s}=\frac{\mathrm{n}_{s} q^{\Delta_{-}}-\mathrm{n}_{s-1} q^{\Delta_{-}^{\prime}}}{(1-q)^{d}}, \quad \Delta_{-}=2-s, \quad \Delta_{-}^{\prime}=1-s
$$

As we shall discuss below, this $\Delta_{+} \rightarrow \Delta_{-}$prescription that was used in the $S^{d}$ case [14] here does not give the full answer: the expression in (1.11) satisfying $\widetilde{\mathcal{Z}}_{-s}(q)=(-1)^{d} \mathcal{Z}_{+s}(1 / q)$ is to be corrected by an extra contribution $\sigma_{s}(q)$ that removes, in particular, all negative powers of $q$ from the small $q$ expansion. This extra term may be interpreted as the character of the finite dimensional irreducible representation of $\mathrm{SO}(d, 2)$ corresponding to the conformal Killing tensors in $d$ dimensions. This is related to gauge degeneracy associated with shadow fields. We shall also provide a general group-theoretic argument for counting of shadow operators based on characters of relevant conformal algebra representations using the general analysis in $[20] .^{7}$

Explicitly, we will find that in $d=4$

$$
\mathcal{Z}_{-s}=\tilde{\mathcal{Z}}_{-s}(q)+\sigma_{s}(q)=\frac{2(2 s+1) q^{2}-(s+1)^{2} q^{s+2}+s^{2} q^{s+3}}{(1-q)^{4}} .
$$

Then, from (1.10) and (1.12),

$$
\mathcal{Z}_{-s}-\mathcal{Z}_{+s}=\frac{2(2 s+1) q^{2}-2(s+1)^{2} q^{s+2}+2 s^{2} q^{s+3}}{(1-q)^{4}} .
$$

This turns out to be the same as the CHS partition function in $d=4$, or, equivalently,

$$
d=4: \quad \mathcal{Z}_{s}=\frac{2 q^{2}\left[(s+1)^{2}\left(1-q^{s}\right)-s^{2}\left(1-q^{s+1}\right)\right]}{(1-q)^{4}},
$$

thus verifying the relations (1.5), (1.7).

Moreover, we will see that one can give a natural interpretation to $\mathcal{Z}_{-s}$ and $\mathcal{Z}_{+s}$ in terms of counting of conformal operators in the CHS theory (1.2) in $\mathbb{R}^{d}$ :

$$
\mathcal{Z}_{-s}=\mathcal{Z}_{s}^{\text {off-shell }}, \quad \mathcal{Z}_{+s}=\mathcal{Z}_{s}^{\text {e.o.m. }}, \quad \mathcal{Z}_{s}=\mathcal{Z}_{s}^{\text {off }- \text { shell }}-\mathcal{Z}_{s}^{\text {e.o.m. }}
$$

Namely, the shadow partition function $\mathcal{Z}_{-s}$ counts (derivatives of) off-shell components of field strength $C_{s}$ (and its derivatives) modulo non-trivial gauge identities while $\mathcal{Z}_{+s}$ counts

\footnotetext{
${ }^{6} \mathrm{Eq} .(1.8)$ has also the interpretation of the character of the short representation of the $\mathrm{SO}(d, 2)$ conformal group with dimension $\Delta_{+}$and spin $s$, i.e. $\chi_{\left(\Delta_{+}, s, 0, \ldots, 0\right)}(q, 1, \ldots, 1)$ which is also a difference of characters of the corresponding long representations $\left(\Delta_{+}, s, 0, \ldots, 0\right)$ and $\left(\Delta_{+}+1, s-1,0, \ldots, 0\right)$ (see [19] and appendix $\mathrm{F}$ below).

${ }^{7}$ For other discussions of shadow fields in the context of AdS/CFT see also [21-24].
} 
the components of the (derivatives of) equations of motion for $C_{s}$ following from the CHS action (1.2) upon variation over $\phi_{s}$ (also modulo identities) that should be subtracted to get the physical on-shell result for $Z_{s}$. The kinematical or off-shell relation between $\mathcal{Z}_{-s}$ and $\mathcal{Z}_{s}$ may not be unexpected as the shadow spin $s$ operators and the conformal higher spins have the same symmetries and dimensions.

For example, in $d=4$ each of the terms in the numerators of (1.12), (1.10) and (1.13) has a clear meaning: (i) $2(2 s+1) q^{2}$ represents the components of the CHS field strength $C_{s}$ of dimension $2\left(6 q^{2}\right.$ for $s=1$ Maxwell field); (ii) $(s+1)^{2} q^{s+2}$ corresponds to the components of the equations of motion tensor $B_{s}=\partial^{s} C_{s}$ and also of the gauge identity tensor $\mathcal{B}_{s}=\left(\epsilon_{4}\right)^{s} \partial^{s} C_{s}$ which are both symmetric traceless rank $s$ tensors of dimension $s+2$ (generalizations of $\partial^{\mu} F_{\mu \nu}$ and $\epsilon^{\mu \nu \lambda \rho} \partial_{\nu} F_{\lambda \rho}$ for $s=1$ ); (iii) $s^{2} q^{s+3}$ term accounts for the trivial identies $\partial B_{s}=0$ and $\partial \mathcal{B}_{s}=0$ (to be added back to avoid overcounting) which are symmetric traceless rank $s-1$ tensors of dimension $s+3$.

The triality relations between different objects and partition functions discussed above are illustrated by the diagram below.

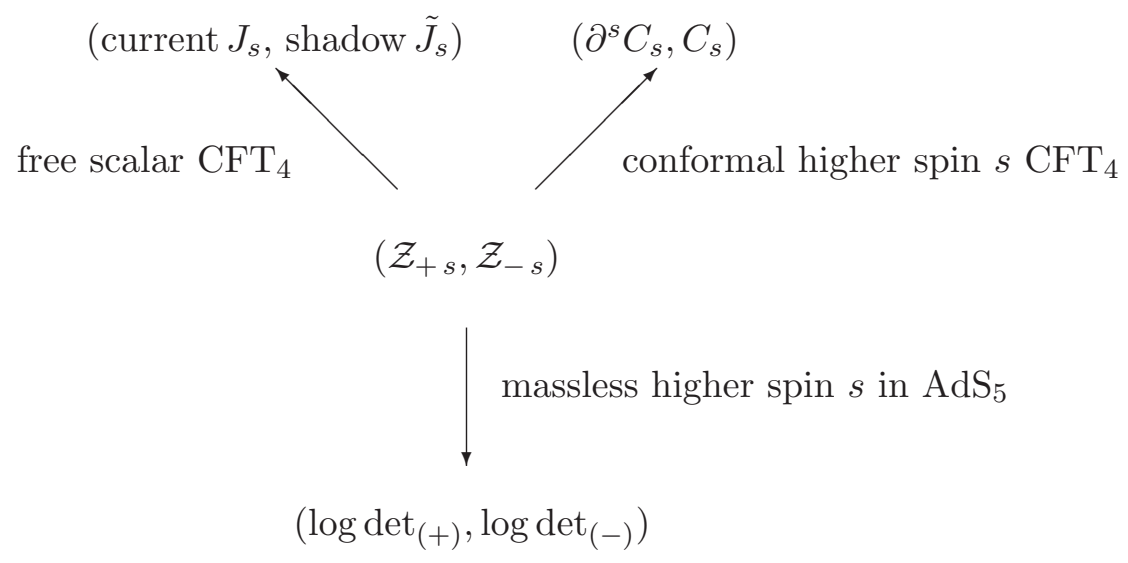

Finally, we may obtain the total CHS partition function by summing over all spins. Assuming a natural regularization discussed in section 6 we find that

$$
d=4: \quad \mathcal{Z}(q)=\sum_{s=0}^{\infty} \mathcal{Z}_{s}(q)=-\frac{q^{2}\left(11+26 q+11 q^{2}\right)}{6(1-q)^{6}}, \quad \mathcal{Z}(q)=\mathcal{Z}(1 / q)
$$

This implies also the vanishing of the associated total Casimir energy on $S^{3}$, as in the case of the massless higher spin partition function $\mathcal{Z}_{+}(q)=\sum_{s=0}^{\infty} \mathcal{Z}_{+s}(q)=\frac{q^{2}(1+q)^{2}}{(1-q)^{6}}$ discussed in [18].

This paper is organized as follows. We shall start in section 2 with a review of the two equivalent methods of computing the canonical partition function of a free CFT: the conformal operator counting method in $\mathbb{R}^{d}[7,8]$ and the finite temperature computation on a spatial sphere $S^{d-1}$. We will illustrate these methods on the examples of standard conformal scalar in $d$ dimensions and Maxwell theory in $d=4$. 
In section 3, we shall generalize the discussion of the spin 1 case to the conformal spin 2 case in $d=4$, i.e. Weyl gravity theory. We shall first compute the corresponding oneloop partition function $\mathcal{Z}_{2}$ on $S^{1} \times S^{3}$ by expanding the non-linear Weyl action (both in 4-derivative and an equivalent 2-derivative formulations) to quadratic order in fluctuations and then check the agreement of the result with the one found by the operator counting method for the Weyl action linearized near flat space.

In section 4 we shall use the above explicit $s=1,2$ results as a motivation for a a proposal for the factorized form of the conformal higher spin $2 s$-derivative operator on the $S^{1} \times S^{3}$ background and for the structure of the associated partition function. We will then support the consistency of our proposal by demonstrating the agreement of the resulting canonical partition function $\mathcal{Z}_{s}$ with the one that can be found directly by counting gauge-invariant conformal operators corresponding to the linearized conformal higher spin action (1.1) in $\mathbb{R}^{4}$.

Next, in section 5.1, we shall clarify the relations (1.7), (1.15) between the conformal spin $s$ partition function and the partition functions associated with spin $s$ conformal operators in the singlet sector of free CFT in $d=4$ and thus also with the one-loop partition functions of massless spin $s$ field in $\mathrm{AdS}_{5}$. We will suggest a method to compute the shadow field partition function $\mathcal{Z}_{-s}(1.12)$ that will allow a straightfoward generalization to $d>4$ (section 5.2) and will thus lead to the expression for the conformal higher spin partition function $\mathcal{Z}_{s}$ generalizing (1.14) to any even dimension $d$. In section 5.3, we shall explain how our general result (5.13) for the conformal higher spin partition function can be derived in a rigorous way in terms of characters of the relevant Verma modules of the conformal algebra $\mathfrak{s o}(d, 2)$ (with details presented in appendix $\mathrm{F}$ ). We shall also comment on the special case of $d=2$.

Finally, in section 6 , we shall sum the individual contributions $\mathcal{Z}_{s}$ to obtain the total (summed over all spins) partition function of the conformal higher spin theory. We shall also find the Casimir energy on $S^{d-1}$ and show that it vanishes if one uses the same regularization prescription that implies the vanishing of the total conformal anomaly $a$ coefficient $[9,10,14,15]$. This happens to be true in any even dimension $d \geq 2$.

Section 7 contains some concluding remarks, empasizing the existence of the full nonlinear generalization of the conformal higher spin action (1.1), (1.2) viewed as an induced action of a free $\mathrm{CFT}_{d}$ in background fields dual to all conserved spin $s$ currents.

There are also several technical appendices. In particular, appendix F (using the results of [20]) explains the structure of spaces of representations and associated characters of the conformal algebra in $d$ dimensions that are relevant for the computation of the CFT partition functions in the main text.

\section{Free CFT partition function: scalar and vector examples}

We shall start with a review of the two equivalent methods of computing the canonical partition function of a free CFT: the operator counting method in $\mathbb{R}^{d}[7,8]$ and the finite temperature computation on a spatial sphere $S^{d-1}$. 
Radial quantization relates conformal operators in $\mathbb{R}^{d}$ with dimensions $\Delta_{n}$ to eigenstates of the Hamiltonian (dilatation operator) on $\mathbb{R}_{t} \times S^{d-1}$. Given the spectrum of eigenvalues $\omega_{n}=\Delta_{n}$ and their degeneracies $\mathrm{d}_{n}$, the "one-particle" or canonical partition function is

$$
\mathcal{Z}(q)=\operatorname{tr} e^{-\beta H}=\sum_{n} \mathrm{~d}_{n} e^{-\beta \omega_{n}}=\sum_{n} \mathrm{~d}_{n} q^{\Delta_{n}}, \quad q \equiv e^{-\beta}
$$

The multi-particle or grand canonical partition function is then given by

$$
\ln Z=-\sum_{n} \mathrm{~d}_{n} \ln \left(1-e^{-\beta \omega_{n}}\right)=\sum_{m=1}^{\infty} \frac{1}{m} \mathcal{Z}\left(q^{m}\right) .
$$

At the same time, (2.2) can be found also from the free QFT path integral on $S_{\beta}^{1} \times S^{d-1}$, i.e. computing the determinant of the corresponding Laplacian $\mathcal{O}$. We shall review the computation of $\mathcal{Z}$ below on the examples of conformal scalar in $d$ dimensions and Maxwell vector in $d=4$. The latter is the first non-trivial representative of the conformal higher spin tower in 4 dimensions.

\subsection{Operator counting method in $\mathbb{R}^{d}$}

Let us start with a free massless scalar field $\Phi$ in $\mathbb{R}^{d}$ with the standard action $\int d^{d} x(\partial \Phi)^{2}$ and thus dimension $\Delta(\Phi)=\frac{1}{2}(d-2)$. The lowest dimension conformal operator is the scalar field itself contributing $q^{\frac{1}{2}(d-2)}$ to the sum in (2.1). Its conformal descendants are found by adding derivatives: $\partial_{\mu_{1}} \ldots \partial_{\mu_{k}} \Phi$. As derivatives in all $d$ dimensions are independent and each power of derivative in a given direction enters only once we get a factor $\sum_{k=0}^{\infty} q^{k}=$ $(1-q)^{-1}$ from each of the $d$ directions. This counting ignores the fact that some operators vanish due to equations of motion $\partial^{2} \Phi=0$. Since $\Delta\left(\partial^{2} \Phi\right)=\frac{1}{2}(d-2)+2$ we thus need to subtract a term $q^{\frac{1}{2}(d-2)+2}$, dressed again by extra derivative factor $(1-q)^{-d}$. The resulting partition function of a conformal scalar is then

$$
\mathcal{Z}_{\text {c.s. }}(q)=\frac{q^{\frac{d-2}{2}}\left(1-q^{2}\right)}{(1-q)^{d}},\left.\quad \mathcal{Z}_{\text {c.s. }}(q)\right|_{d=4}=\frac{q-q^{3}}{(1-q)^{4}}
$$

This one-particle partition function corresponds to the character of the free scalar (Dirac singleton) representation of the conformal group $\mathrm{SO}(d, 2)$ (see [19]).

Next, let us consider the standard Maxwell vector field in $d=4$. Here lowest dimension gauge-invariant operator is the field strength $F_{\mu \nu}$ with dimension $\Delta=2$ and 6 components, giving a term $6 q^{2}$. Its derivatives give extra factor of $(1-q)^{-4}$. This overcounts by ignoring the vanishing of some operators due to equations of motion $\partial^{\mu} F_{\mu \nu}=0$ and gauge identities $\partial^{\mu} F_{\mu \nu}^{*}=0$ (and their derivatives). This suggests subtraction of $-(4+4) q^{3}$ times $(1-q)^{-4}$, but this also overcounts as some identites are trivial, descending from the two $\partial_{\mu} \partial_{\nu} F^{\mu \nu}=0$ and $\partial^{\mu} \partial^{\nu} F_{\mu \nu}^{*}=0$ corresponding to operators of dimension 4 . This requires adding back $2 q^{4}(1-q)^{-4}$. The final $d=4$ vector partition function is then

$$
\mathcal{Z}_{1}(q)=\frac{6 q^{2}-8 q^{3}+2 q^{4}}{(1-q)^{4}}=\frac{2(3-q) q^{2}}{(1-q)^{3}}
$$


This counting argument can be straightfowardly generalized to the case of a conformal vector in even $d$ dimensions with the action (1.2), i.e. $S_{1}=-\frac{1}{4} \int d^{d} x F_{\mu \nu} \partial^{d-4} F_{\mu \nu}$ (see section 6 below).

\subsection{Partition function on $S^{1} \times S^{d-1}$}

Let us now review how the same expressions for $\mathcal{Z}$ can be found by computing the standard QFT partition function on a curved $S_{\beta}^{1} \times S^{d-1}$ background (we assume that $S^{d-1}$ has unit radius). The partition function of a free conformally coupled scalar is

$$
-\log Z_{\text {c.s. }}=\frac{1}{2} \log \operatorname{det} \mathcal{O}_{0}, \quad \mathcal{O}_{0}=-D^{2}+\frac{d-2}{4(d-1)} R .
$$

On $S^{1} \times S^{d-1}$ we have

$$
D^{2} \equiv D_{\mu} D^{\mu}=\partial_{0}^{2}+\mathbf{D}^{2}, \quad \mathbf{D}^{2}=\mathbf{D}^{i} \mathbf{D}_{i}=D_{S^{d-1}}^{2}
$$

where $\partial_{0}$ is derivative along compact euclidean time direction of length $\beta$. The scalar curvature is $R=R\left(S^{d-1}\right)=(d-1)(d-2)$, so that

$$
\mathcal{O}_{0}=-\partial_{0}^{2}-\mathbf{D}^{2}+\frac{1}{4}(d-1)^{2} .
$$

The eigenvalues of the Laplacian $-\mathbf{D}^{2}$ on $S^{d-1}$ and their multiplicities are (see appendix A)

$$
\begin{aligned}
& \lambda_{n}\left(S^{d-1}\right)=n(n+d-2), \\
& \mathrm{d}_{n}\left(S^{d-1}\right)=(2 n+d-2) \frac{(n+d-3) !}{n !(d-2) !} .
\end{aligned}
$$

Hence, the eigenvalues of (2.7) are

$$
\lambda_{k, n}=w^{2}+\omega_{n}^{2}, \quad w=\frac{2 \pi k}{\beta}, \quad \omega_{n}=n+\frac{1}{2}(d-2), \quad k \in \mathbb{Z}, \quad n=0,1,2, \ldots
$$

Then, computing $-\ln Z_{\text {c.s. }}=\frac{1}{2} \log \operatorname{det} \mathcal{O}_{0}=\frac{1}{2} \sum_{k, n} \mathrm{~d}_{n} \log \lambda_{k, n}$ one finds by the standard argument the expression in (2.2) where

$$
\mathcal{Z}_{\text {c.s. }}(\beta)=\sum_{n=0}^{\infty} d_{n} e^{-\beta\left[n+\frac{1}{2}(d-2)\right]}=\frac{q^{\frac{d-2}{2}}\left(1-q^{2}\right)}{(1-q)^{d}},
$$

which is indeed the same as in (2.3).

The quantization of the $d=4$ Maxwell action $S_{1}=-\frac{1}{4} \int d^{4} x \sqrt{g} F_{\mu \nu} F^{\mu \nu}$ in curved background in covariant Lorentz gauge gives the following well-known expression for the vector field partition function

$$
Z_{1}=\frac{\operatorname{det}\left(-D^{2}\right)}{\left[\operatorname{det}\left(-g_{\mu \nu} D^{2}+R_{\mu \nu}\right)\right]^{1 / 2}},
$$

Specializing to $S^{1} \times S^{3}$ where $R_{00}=0, \quad R_{i j}=\frac{1}{3} g_{i j} R, R=6$, and $A_{\mu}=\left(A_{0}, A_{i}\right) \quad(i, j=$ $1,2,3)$ we get from $(2.12)$

$$
Z_{1}=\left[\frac{\operatorname{det}\left(-D^{2}\right)}{\operatorname{det}\left(-g_{i j} D^{2}+R_{i j}\right)}\right]^{1 / 2}
$$


Splitting the 3 -vector field operator into the transverse $\left(D^{i} A_{i, \perp}=0\right)$ and longitudinal parts we end up with (using that $R_{i j}=2 g_{i j}$ )

$$
Z_{1}=\frac{1}{\left[\operatorname{det}\left(-g_{i j} D^{2}+R_{i j}\right)_{\perp}\right]^{1 / 2}}=\frac{1}{\left[\operatorname{det} \mathcal{O}_{1 \perp}\right]^{1 / 2}}, \quad \mathcal{O}_{1 i j}=\left(-\partial_{0}^{2}-\mathbf{D}^{2}+2\right)_{i j}
$$

where $\mathcal{O}_{1 \perp}$ is defined on transverse 3 -vectors.

The same expression can be obtained directly by choosing the temporal gauge $A_{0}=0$ in the original path integral. In $S^{1} \times S^{3}$ case the corresponding ghost factor is $\operatorname{det}\left(\partial_{0}\right)$ while the Lagrangian is $\mathscr{L}=-\frac{1}{4} F_{\mu \nu} F^{\mu \nu}=-\frac{1}{2} \partial_{0} A_{i} \partial_{0} A^{i}-\frac{1}{4} F_{i j} F^{i j}$. Changing variables $A_{i}=A_{i \perp}+D_{i} \varphi$ introduces the Jacobian factor $\left[\operatorname{det}\left(-\mathbf{D}^{2}\right)\right]^{1 / 2}$ while the Lagrangian becomes (up to a total derivative)

$$
\mathscr{L}=-\frac{1}{2} \varphi \partial_{0}^{2} \mathbf{D}^{2} \varphi-\frac{1}{2} A_{\perp}^{i}\left(-g_{i j} D^{2}+R_{i j}\right) A_{\perp}^{j}
$$

Integration over $\varphi$ gives the contribution $\left[\operatorname{det}\left(-\partial_{0}^{2} \mathbf{D}^{2}\right)\right]^{-1 / 2}$ that cancels the product of the ghost and Jacobian factors. The final result is thus again (2.14).

Using the eigenvalues and their multiplicities of the transverse vector Laplacian $\left(-\mathbf{D}^{2}\right)_{1 \perp}$ on $S^{3}$ given by (A.1)-(A.4), we conclude that the spectrum of $\mathcal{O}_{1 \perp}$ in $(2.14)$ is $\left(\partial_{0} \rightarrow i w, w=\frac{2 \pi k}{\beta}\right.$, cf. $\left.(2.10)\right)$

$$
\lambda_{k, n}=w^{2}+\left(n^{2}+4 n+2\right)+2=w^{2}+\omega_{n}^{2}, \quad \omega_{n}=n+2, \quad \mathrm{~d}_{n}=2(n+1)(n+3),
$$

and thus the one-particle partition function corresponding to $Z_{1}$ in (2.14) is given by

$$
\mathcal{Z}_{1}(\beta)=\sum_{n=0}^{\infty} \mathrm{d}_{n} e^{-\beta(n+2)}=\frac{2(3-q) q^{2}}{(1-q)^{3}}
$$

This is again in agreement with the expression (2.4) found by the operator counting method.

\section{Conformal spin 2 in $d=4$}

Let us now consider the conformal spin 2 case in $d=4$, i.e. Weyl gravity with the full non-linear action being (we drop total derivative)

$$
S_{2}=\frac{1}{2} \int d^{4} x \sqrt{g} C_{\mu \nu \lambda \rho} C^{\mu \nu \lambda \rho}=\int d^{4} x \sqrt{g}\left(R_{\mu \nu} R_{\mu \nu}-\frac{1}{3} R^{2}\right)
$$

Here, we shall first compute the corresponding one-loop partition function on $S^{1} \times S^{3}$ by expanding the action (3.1) to quadratic order in fluctuations near this conformally flat background. We shall then check the agreement of the result with the one found by the operator counting method for the linearized action (3.1) expanded near $\mathbb{R}^{4}$. 


\subsection{Quadratic fluctuation operator in conformally flat background}

Since we are interested in quantizing spin 2 fluctuations on the conformally flat $S^{1} \times$ $S^{3}$ background, ${ }^{8}$ in expanding (3.1) we may ignore terms with the Weyl tensor of the background metric. We may also drop terms with covariant derivatives of the curvature. Then, using the expressions in appendix B and assuming the reparametrization and Weyl gauge conditions $D_{\mu} h^{\mu \nu}=0, h_{\mu}^{\mu}=0$, we find from (3.1) the following quadratic fluctuation Lagrangian

$$
\begin{aligned}
\mathscr{L}^{(2)}= & \frac{1}{4} D^{2} h_{\mu \nu} D^{2} h^{\mu \nu}-R_{\rho}^{\mu} h_{\mu \nu} D^{2} h^{\nu \rho}+\frac{1}{2} R^{\mu \nu} h_{\alpha \beta} D_{\mu} D_{\nu} h^{\alpha \beta} \\
& -\frac{3}{2} R_{\rho \sigma} R^{\sigma \mu} h_{\mu \nu} h^{\nu \rho}+\frac{1}{2} R^{\nu \rho} R^{\sigma \mu} h_{\mu \nu} h_{\rho \sigma}+\frac{1}{6}\left(h_{\mu \nu} R^{\mu \nu}\right)^{2}+\frac{1}{4} R_{\mu \nu} R^{\mu \nu} h_{\alpha \beta} h^{\alpha \beta} \\
& +\frac{1}{2} R R_{\rho}^{\mu} h_{\mu \nu} h^{\nu \rho}-\frac{1}{9} R^{2} h_{\mu \nu} h^{\mu \nu} .
\end{aligned}
$$

In the special case when our conformally flat background is also an Einstein space $R_{\mu \nu}=$ $\frac{1}{4} R g_{\mu \nu}$, i.e. for $S^{4}$ or $\mathrm{AdS}_{4}$, the Lagrangian (3.2) reduces to

$$
\mathscr{L}_{R_{\mu \nu}=\frac{1}{4} R g_{\mu \nu}}^{(2)}=\frac{1}{4} D^{2} h_{\mu \nu} D^{2} h^{\mu \nu}-\frac{1}{8} R h_{\mu \nu} D^{2} h^{\mu \nu}+\frac{1}{72} R^{2} h_{\mu \nu} h^{\mu \nu}=\frac{1}{4} h^{\mu \nu} \tilde{\mathcal{O}}_{2} h_{\mu \nu}
$$

where the 4-th order operator $\mathcal{O}$ defined on transverse traceless tensors $h_{\mu \nu}$ takes the factorized form [25-28]

$$
\tilde{\mathcal{O}}_{2}=\left(-D^{2}+\frac{1}{6} R\right)\left(-D^{2}+\frac{1}{3} R\right)
$$

or $\tilde{\mathcal{O}}_{2}=\left(-D^{2}+2\right)\left(-D^{2}+4\right)$ for a unit-radius $S^{4}$ with $R=12$.

To analyse the non-Einstein case of $S^{1} \times S^{3}$ background, let us split the components of $h_{\mu \nu}$ into $h_{i j}, h_{0 i}, h_{00}$ and use that here $R_{00}=0, R_{i j}=\frac{1}{3} R g_{i j}, R=6$. Then, $h_{i j}$ decouples from $h_{0 i}$ and $h_{00}$ in (3.2), with the transverse traceless $h_{i j}$ dependent part being

$$
\mathscr{L}_{S^{1} \times S^{3}}^{(2)}=\frac{1}{4} D^{2} h_{i j} D^{2} h^{i j}-\frac{1}{3} R h_{i j} \partial_{0}^{2} h^{i j}-\frac{1}{6} R h_{i j} \mathbf{D}^{2} h^{i j}+\frac{1}{36} R^{2} h_{i j} h^{i j},
$$

where we used the notation in (2.6). The corresponding 4-th order operator is thus

$$
\mathcal{O}_{2}=\left(\partial_{0}^{2}+\mathbf{D}^{2}\right)^{2}-\frac{2}{3} R\left(2 \partial_{0}^{2}+\mathbf{D}^{2}\right)+\frac{1}{9} R^{2} .
$$

It is useful to rederive this expression in the 2nd-derivative formulation of conformal higher spin theory involving auxiliary fields $[29,30]$. In the spin 2 case the corresponding Lagrangian may be written as $[29,31]$

$$
\mathscr{L}(g, f)=\sqrt{g}\left[-f^{\mu \nu} G_{\mu \nu}-\frac{1}{4} f^{\mu \nu} f_{\mu \nu}+\frac{1}{4}\left(g^{\mu \nu} f_{\mu \nu}\right)^{2}\right], \quad G_{\mu \nu} \equiv R_{\mu \nu}-\frac{1}{2} g_{\mu \nu} R .
$$

\footnotetext{
${ }^{8}$ This background solves the Bach equations of motion corresponding to (3.1) so that the resulting partition function will be gauge-independent.
} 
Solving for the auxiliary symmetric tensor $f_{\mu \nu}$ we get back to the Weyl action (3.1). Expanding around a generic curved background for $g_{\mu \nu}$ with $f_{\mu \nu}=-2\left(R_{\mu \nu}-\frac{1}{6} g_{\mu \nu} R\right)$ it is straightforward to find the corresponding quadratic fluctuation action for $\delta f_{\mu \nu}=\phi_{\mu \nu}$ and $\delta g_{\mu \nu}=h_{\mu \nu}$. Assuming gauge conditions of transversality and tracelessness of $h_{\mu \nu}$ and ignoring terms involving Weyl tensor and derivatives of the curvature (as we are interested in a conformally flat constant-curvature background), we find ${ }^{9}$

$$
\begin{aligned}
\mathscr{L}^{(2)} & =\mathscr{L}_{\phi \phi}^{(2)}+\mathscr{L}_{\phi h}^{(2)}+\mathscr{L}_{h h}^{(2)}, \\
\mathscr{L}_{\phi \phi}^{(2)} & =-\frac{1}{4} \phi_{\alpha \beta} \phi^{\alpha \beta}, \quad \mathscr{L}_{\phi h}^{(2)}=\frac{1}{6} R h^{\alpha \beta} \phi_{\alpha \beta}-\phi^{\alpha \beta} D_{\gamma} D_{\beta} h_{\alpha}^{\gamma}+\frac{1}{2} \phi^{\alpha \beta} D^{2} h_{\alpha \beta}, \\
\mathscr{L}_{h h}^{(2)}= & -\frac{1}{4}\left(R_{\gamma \delta} R^{\gamma \delta}-\frac{1}{3} R^{2}\right) h^{\alpha \beta} h_{\alpha \beta}+2 R_{\alpha \gamma} R_{\beta \delta} h^{\alpha \beta} h^{\gamma \delta}+R_{\alpha \beta} R_{\gamma \delta} h^{\alpha \beta} h^{\gamma \delta} \\
& -3 R_{\beta}^{\delta} R_{\gamma \delta} h_{\alpha}^{\gamma} h^{\alpha \beta}+R_{\gamma \delta} R^{\gamma \delta} h_{\alpha \beta} h^{\alpha \beta}+3 R_{\beta \gamma} R h_{\alpha}^{\gamma} h^{\alpha \beta}-\frac{19}{36} R^{2} h_{\alpha \beta} h^{\alpha \beta} \\
& +\frac{1}{2} R^{\alpha \beta} D_{\alpha} h^{\gamma \delta} D_{\beta} h_{\gamma \delta}+R^{\gamma \delta} h^{\alpha \beta} D_{\beta} D_{\alpha} h_{\gamma \delta}-\frac{5}{6} R h^{\alpha \beta} D^{2} h_{\alpha \beta}+\frac{1}{3} R D_{\beta} h_{\alpha \gamma} D^{\gamma} h^{\alpha \beta} \\
& -\frac{1}{2} R D_{\gamma} h_{\alpha \beta} D^{\gamma} h^{\alpha \beta}-2 R^{\gamma \delta} h^{\alpha \beta} D_{\delta} D_{\beta} h_{\alpha \gamma}+R^{\gamma \delta} h^{\alpha \beta} D_{\delta} D_{\gamma} h_{\alpha \beta}+2 R_{\alpha}^{\gamma} h^{\alpha \beta} D^{2} h_{\beta \gamma} \\
& -R^{\alpha \beta} D_{\gamma} h_{\beta \delta} D^{\delta} h_{\alpha}^{\gamma}+R^{\alpha \beta} D_{\delta} h_{\beta \gamma} D^{\delta} h_{\alpha}^{\gamma},
\end{aligned}
$$

where the first term in (3.10) comes from the expansion of the $\sqrt{g}$ factor in (3.7). Specializing to the case of the $S^{1} \times S^{3}$ background and concentrating on the part of the action that depends on the transverse traceless spatial parts of the fluctuations $h_{i j}$, $\phi_{i j}$, we get (after commuting derivatives, integrating by parts and using that $R_{00}=0, R_{i j}=\frac{1}{3} R g_{i j}$ )

$$
\mathscr{L}_{\phi h}^{(2)}=\frac{1}{2} \phi^{i j}\left(D^{2}-\frac{2}{3} R\right) h_{i j}, \quad \mathscr{L}_{h h}^{(2)}=\frac{1}{6} R h^{i j}\left(\mathbf{D}^{2}-\frac{1}{2} R\right) h_{i j},
$$

so that (3.8) becomes

$$
\mathscr{L}^{(2)}=\frac{1}{2} \phi^{i j}\left(D^{2}-\frac{2}{3} R\right) h_{i j}-\frac{1}{4} \phi_{i j} \phi^{i j}+\frac{1}{6} R h^{i j}\left(\mathbf{D}^{2}-\frac{1}{2} R\right) h_{i j} .
$$

Note that the kinetic $h$ term is absent in the Lagrangian (3.7) expanded near flat space but it appears on a curved background. Using that $R=6$ (for a unit-radius $S^{3}$ ), eq. (3.12) can be written also as

$$
\mathscr{L}^{(2)}=\frac{1}{2} \phi^{i j}\left(D^{2}-4\right) h_{i j}-\frac{1}{4} \phi_{i j} \phi^{i j}+h^{i j}\left(\mathbf{D}^{2}-3\right) h_{i j} .
$$

Solving for $\phi_{i j}$, we then find that $\mathscr{L}^{(2)}=\frac{1}{4} h^{i j} \mathcal{O}_{2} h_{i j}$ where

$$
\mathcal{O}_{2}=\left(D^{2}-4\right)^{2}+4\left(\mathbf{D}^{2}-3\right)=\partial_{0}^{4}+2 \partial_{0}^{2}\left(\mathbf{D}^{2}-4\right)+\left(\mathbf{D}^{2}-2\right)^{2},
$$

is indeed equivalent to (3.6). Note that $\mathcal{O}_{2}$ can be written in the following factorized form

$$
\mathcal{O}_{2}=\left[\left(\partial_{0}-1\right)^{2}+\mathbf{D}^{2}-3\right]\left[\left(\partial_{0}+1\right)^{2}+\mathbf{D}^{2}-3\right],
$$

which is the $S^{1} \times S^{3}$ counterpart of (3.4) found in $S^{4}$ or $\mathrm{AdS}_{4}$ case (where $R= \pm 12$ ).

\footnotetext{
${ }^{9}$ The case of a generic Einstein background where the fluctuation operator also factorizes is discussed in appendix C.
} 


\subsection{Partition function on $S^{1} \times S^{3}$}

Like in the vector field case in section (2.2), the derivation of the one-loop partition function can be presented either in 4-d covariant gauge or in the time-like reparametrization gauge $h_{00}=0, h_{0 i}=0$ with the Weyl gauge $h_{\mu}^{\mu}=0$ being then equivalent to the tracelessness of $h_{i j}$. Splitting $h_{i j}$ into the transverse and longitudinal parts (and taking into account various ghost and Jacobian factors as in the vector field case discussed in section 2.2), we end up with the following simple expression for the spin 2 analog of (2.14):

$$
Z_{2}=\frac{1}{\left[\operatorname{det} \mathcal{O}_{2 \perp} \operatorname{det}^{\prime} \mathcal{O}_{1 \perp}\right]^{1 / 2}} .
$$

Here the spin 2 operator $\mathcal{O}_{2} \perp$ given in (3.6), (3.14) is defined on transverse traceless tensors $h_{i j}$ while the spin 1 one is the same as in (2.14). ${ }^{10}$ The vector determinant defined on transverse vector $V_{i}$ originates from the decomposition $h_{i j} \rightarrow h_{i j}^{\perp}+D_{i} V_{j}+D_{j} V_{i}, D^{i} V_{i}=0$. The prime indicates that the lowest $n=0$ mode of the vector Laplacian on $S^{3}$ is to be dropped since this mode satisfies $D_{i} V_{j}+D_{j} V_{i}=0$ and thus cannot appear from $h_{i j}$ (see appendix D).

The spectrum of $\mathcal{O}_{1} \perp$ was already given in (2.16). Using that the spectrum of the transverse traceless rank 2 tensor Laplacian on $S^{3}$ can be read off from (A.2)-(A.3), i.e.

$$
\left(-\mathbf{D}^{2}\right)_{2 \perp}: \quad \lambda_{n}=(n+2)(n+4)-2, \quad \mathrm{~d}_{n}=2(n+1)(n+5), \quad n=0,1,2, \ldots,
$$

we conclude that the eigenvalues of $\mathcal{O}_{2} \perp$ in $(3.14)$ can be written as $\left(\partial_{0}^{2} \rightarrow-w^{2}\right.$, cf. $(2.10),(2.16))$

$$
\begin{aligned}
\mathcal{O}_{2 \perp}: \quad \quad \quad \lambda_{k, n} & =w^{4}+2 w^{2}[(n+2)(n+4)+2]+[(n+2)(n+4)]^{2} \\
& =\left[w^{2}+(n+2)^{2}\right]\left[w^{2}+(n+4)^{2}\right] .
\end{aligned}
$$

This simple result is related to the special structure of $\mathcal{O}_{2}$ in (3.14), (3.15): its eigenvalues factorize with the spatial parts being squares of the effective energies $\omega_{n}$ which are linear in $n$ as in the conformal scalar (2.10) and vector (2.16) cases. This is also a consequence of the underlying conformal invariance of the spin 2 theory.

As a result, the conformal spin 2 partition function takes the standard form (2.2) where the canonical partition function is a combination of the two terms corresponding to the two factors in (3.16)

$$
\mathcal{Z}_{2}=\mathcal{Z}_{2,0}(q)+\mathcal{Z}_{1,1}(q)
$$

The notation $\mathcal{Z}_{s, r}$ means that the sum over $n$ starts with $n=r$, so that the explict expressions following from (3.18) and (2.16), (2.17) are

$$
\begin{aligned}
& \mathcal{Z}_{2,0}(q)=\sum_{n=0}^{\infty} 2(n+1)(n+5)\left(q^{n+2}+q^{n+4}\right), \\
& \mathcal{Z}_{1,1}(q)=\sum_{n=1}^{\infty} 2(n+1)(n+3) q^{n+2} .
\end{aligned}
$$

\footnotetext{
${ }^{10}$ Note that this combination of determinants describes the right number of degrees of freedom a conformal graviton: $(6-1-1)+2=6$.
} 
Doing the sums gives finally

$$
\mathcal{Z}_{2}=\frac{10 q^{2}-18 q^{4}+8 q^{5}}{(1-q)^{4}}=\frac{2 q^{2}\left(5+5 q-4 q^{2}\right)}{(1-q)^{3}} .
$$

\subsection{Partition function from conformal operator counting in $\mathbb{R}^{4}$}

Let us now show that the same expression (3.22) for the conformal spin 2 partition function can be found in the operator counting method, i.e. by treating the linearized Weyl gravity (3.1) as a CFT in $\mathbb{R}^{4}$ and counting gauge-invariant conformal operators built out of the linearized Weyl tensor $C \sim \partial \partial h$ weighted with their conformal dimension and subtracting the contributions of gauge identities and equations of motion.

As in the conformal scalar and $d=4$ vector examples discussed in section 2.1, adding all possible derivatives introduces the universal overall denominator factor $(1-q)^{4}$, so the main problem is to determine the numerator. First, let us count the off-shell components of $C_{\mu_{1} \nu_{1} \mu_{2} \nu_{2}}$ modulo gauge identities. $C$ has dimension 2 ( $h$ has dimension 0 ) and 10 independent components (transforming as the $(2,2)$ representation of $\mathrm{SO}(4)$ ); this gives $10 q^{2}$ contribution. Adding all possible derivatives to $C$ produces overcounting since there are non-trivial gauge identities that $C \sim \partial \partial h$ satisfies, i.e.

$$
\mathcal{B}^{\mu_{1} \mu_{2}} \equiv \varepsilon^{\mu_{1} \nu_{1} \gamma_{1} \delta_{1}} \varepsilon^{\mu_{2} \nu_{2} \gamma_{2} \delta_{2}} \partial_{\nu_{1}} \partial_{\nu_{2}} C_{\gamma_{1} \delta_{1} \gamma_{2} \delta_{2}}=0
$$

and their derivatives. $\mathcal{B}^{\mu \nu}$ has dimension 4 and is symmetric and traceless with 9 components, i.e. this requires subtracting the $9 q^{4}$ term. However, subtracting all the derivatives of $\mathcal{B}^{\mu \nu}$ would also overcount as $\mathcal{B}^{\mu \nu}$ itself satisfies the identity $\partial_{\mu} \mathcal{B}^{\mu \lambda}=0$ which has dimension 5 and 4 components; this requires adding back $4 q^{5}$. Thus, the off-shell count of the components of the Weyl tensor and its derivatives leads to the following contribution to the partition function (2.1)

$$
\mathcal{Z}_{2}^{\text {off }- \text { shell }}=\frac{10 q^{2}-9 q^{4}+4 q^{5}}{(1-q)^{4}} .
$$

It remains to subtract also some of the descendant operators $\partial \ldots \partial C$ that vanish due to the equations of motion for the dynamical field $h_{\mu \nu}$, i.e.

$$
B_{\mu_{1} \mu_{2}} \equiv \partial^{\nu_{1}} \partial^{\nu_{2}} C_{\mu_{1} \nu_{1} \mu_{2} \nu_{2}}=0 \text {. }
$$

The count of the symmetric traceless $B_{\mu_{1} \mu_{2}}$ is the same as for the $\mathcal{B}^{\mu_{1} \mu_{2}}$ above. We need to subtract $9 q^{4}$ but also to add back $4 q^{5}$ to account for the identity $\partial^{\mu} B_{\mu \lambda}=0$. Thus the contribution of the equations of motion that should be subtracted from (3.24) is

$$
\mathcal{Z}_{2}^{\text {e.o.m. }}=\frac{9 q^{4}-4 q^{5}}{(1-q)^{4}}
$$

As a result,

$$
\mathcal{Z}_{2}=\mathcal{Z}_{2}^{\text {off }- \text { shell }}-\mathcal{Z}_{2}^{\text {e.o.m. }}=\frac{10 q^{2}-2\left(9 q^{4}-4 q^{5}\right)}{(1-q)^{4}}
$$

is indeed the same as (3.22). 
Let us note that as in the $s=1$ case in section 2.1 here (i) the contributions of the equations of motion and of the gauge identities are the same, i.e. they just double, and (ii) the count of the equations of motion contribution is the same as the count of the conserved traceless rank $s$ "current" operator of dimension $2+s$ (conformal stress tensor for $s=2$ ). As we shall discuss in the next section, these features generalize to any spin $s$ conformal field case in $d=4$.

\section{Partition function of general conformal higher spin field in $d=4$}

Let us now use the explicit $s=1,2$ results found above as a motivation for the form of the conformal higher spin operator and the structure of the associated partition function on the $S^{1} \times S^{3}$ background. We will then demonstrate the agreement of the resulting canonical partition function $\mathcal{Z}_{s}$ with the one found directly by counting gauge-invariant conformal operators corresponding to the linearized conformal higher spin action (1.1) in $\mathbb{R}^{4}$.

Namely, we shall assume that the $2 s$-derivative conformal spin $s$ operator, evaluated on the $S^{1} \times S^{3}$ background and restricted to transverse traceless spin $s>0$ tensors with 3-dimensional indices $\phi_{i_{1} \ldots i_{s}}$, takes the following form:

$$
\begin{array}{ll}
s=\text { even }: & \mathcal{O}_{s}=\prod_{p=1}^{s}\left[\left(\partial_{0}+2 p-s-1\right)^{2}+\mathbf{D}^{2}-s-1\right], \\
s=\text { odd }: & \mathcal{O}_{s}=-\prod_{p=-\frac{s-1}{2}}^{\frac{s-1}{2}}\left[\left(\partial_{0}+2 p\right)^{2}+\mathbf{D}^{2}-s-1\right] .
\end{array}
$$

For example, for $s=1$ we get $\mathcal{O}_{1}$ in (2.14), for $s=2$ we find $\mathcal{O}_{2}$ in (3.15), while for $s=3$ eq. (4.2) gives $\mathcal{O}_{3}=\left(\partial_{0}^{2}+\mathbf{D}^{2}-4\right)\left[\left(\partial_{0}+2\right)^{2}+\mathbf{D}^{2}-4\right]\left[\left(\partial_{0}-2\right)^{2}+\mathbf{D}^{2}-4\right]$, etc. Eqs. $(4.1)$ and (4.2) may be viewed is the $S^{1} \times S^{3}$ counterpart of the factorized form [9, 11, 12] of the $\mathcal{O}_{s}$ operator on $S^{4}$ or $\mathrm{AdS}_{4}$ background. ${ }^{11}$

Using that the spectrum of Laplacian $-\mathbf{D}^{2}$ on $S^{3}$ is given by (see (A.1)-(A.2))

$$
\left(-\mathbf{D}^{2}\right)_{s \perp}: \quad \lambda_{n}=(n+s)(n+s+2)-s, \quad \mathrm{~d}_{n}=2(n+1)(n+2 s+1),
$$

we then find that the eigenvalues of $\mathcal{O}_{s \perp}$ may be written in a simple form generalizing (2.16) and (3.18) $\left(w=2 \pi k \beta^{-1}\right)$

$$
\lambda_{k, n}=\prod_{p=1}^{s}\left(w^{2}+\omega_{n, p}^{2}\right), \quad \omega_{n, p}=n+2 p .
$$

\footnotetext{
${ }^{11}$ For spin $s \geq 2$, a derivation of the factorization (4.1), (4.2) from the quadratic expansion of a curved space action, as in section 3 for $s=2$, is not possible because a general form of a CHS action in curved space is not yet available (though one should be able to derive it in a general conformally-flat background as in the AdS case in $[11,12])$. Nevertheless, one may use a heuristic approach by trying to generalize the 2nd-derivative formulation of conformal higher spin theory in flat background developed in [29, 30] to $S^{1} \times S^{3}$ background. For example, for spin 3, one can assume that, like in the $s=2$ case (3.13), the (gauge-fixed) Lagrangian should be a quadratic form in three auxiliary symmetric traceless fields $\phi_{i j k}^{(1,2,3)}$ whose coefficients are linear combinations of covariant derivatives $\partial_{0}$ and $\mathbf{D}^{2}$ allowed by dimension counting. Requiring that such a Lagrangian $\mathscr{L}_{3}^{(2)}$ leads to the factorized operator $\mathcal{O}_{s}$ does not fix it completely, but imposes many constraints. One natural solution is $\mathscr{L}_{3}^{(2)}=\phi^{(1)}\left(\partial_{0}^{2}+\mathbf{D}^{2}-4\right) \phi^{(3)}+\phi^{(2)}\left(\partial_{0}^{2}+\mathbf{D}^{2}+4\right) \phi^{(2)}-$ $\phi^{(2)} \phi^{(3)}-16 \phi^{(2)}\left(\partial_{0}^{2}-1\right) \phi^{(1)}$.
} 
Our proposal for the corresponding partition function generalizing (2.14), (3.16) is then

$$
Z_{s}=\frac{1}{\left[\prod_{p=1}^{s} \operatorname{det}^{\prime} \mathcal{O}_{p \perp}\right]^{1 / 2}},
$$

where prime in $\operatorname{det}^{\prime} \mathcal{O}_{p \perp}$ means that the product of the eigenvalues should start with $n=s-p$, i.e. first $s-p$ modes are to be omitted. As in the spin 2 case, this is related to the fact that these modes do not appear in the transverse traceless decomposition of the symmetric traceless tensor $\phi_{i_{1} \ldots i_{s}}$ (see appendix D).

The canonical partition function corresponding to (4.5) can then be written as

$$
\mathcal{Z}_{s}=\sum_{p=1}^{s} \mathcal{Z}_{p, s-p}=\mathcal{Z}_{s, 0}+\mathcal{Z}_{s-1,1}+\cdots+\mathcal{Z}_{1, s-1}
$$

where $\mathcal{Z}_{p, s-p}$ is the contribution of spin $p$ operator in (4.5) following from (4.4)

$$
\begin{aligned}
\mathcal{Z}_{p, s-p} & =\sum_{n=s-p}^{\infty} 2(n+1)(n+2 p+1) \sum_{r=1}^{p} q^{n+2 r} \\
& =\frac{2 q^{s+2-p}\left(1-q^{2 p}\right)\left[1+q+\left(s^{2}-p^{2}\right)(1-q)^{2}+2 s(1-q)\right]}{(1+q)(1-q)^{4}} .
\end{aligned}
$$

Performing the sum in (4.6) we then finish with

$$
\begin{aligned}
\mathcal{Z}_{s}(q) & =\frac{2 q^{2}}{(1-q)^{4}}\left[(s+1)^{2}\left(1-q^{s}\right)-s^{2}\left(1-q^{s+1}\right)\right] \\
& =\frac{2(2 s+1) q^{2}-2(s+1)^{2} q^{s+2}+2 s^{2} q^{s+3}}{(1-q)^{4}}
\end{aligned}
$$

This generalizes the above $s=1(2.4)$ and $s=2(3.22)$ expressions to any $s>2$.

Let us now generalize the discussion in section 3.3 to $s>2$ to demonstrate that the same expression (4.8) follows indeed from the operator counting method. The generalized Weyl tensor $C_{\mu_{1} \nu_{1} \ldots \mu_{s} \nu_{s}}$ in (1.1), (1.2) transforms in the $(s, s, 0, \ldots, 0)$ representation of $\mathrm{SO}(d)$ corresponding to the rectangular Young tableau with two rows and $s$ columns

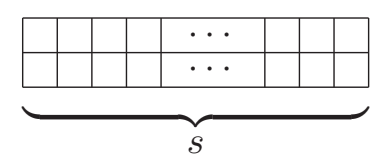

Its dimension is given by (see, e.g., [32])

$$
\begin{aligned}
\mathrm{n}_{(s, s)} & =\frac{(2 s+d-4)(2 s+d-3)(2 s+d-2)(s+d-5) !(s+d-4) !}{s !(s+1) !(d-2) !(d-4) !}, \\
\left.\mathrm{n}_{(s, s)}\right|_{d=4} & =2(2 s+1) .
\end{aligned}
$$

Since $C_{s}$ has dimension 2 , its components contribute $2(2 s+1) q^{2}$ to the numerator of $\mathcal{Z}_{s}$. The non-trivial gauge identities that generalize (3.23) (and which are implicitly contained in the general discussion in [5]) are

$$
\mathcal{B}^{\mu_{1} \cdots \mu_{s}} \equiv \varepsilon^{\mu_{1} \nu_{1} \gamma_{1} \delta_{1}} \cdots \varepsilon^{\mu_{s} \nu_{s} \gamma_{s} \delta_{s}} \partial_{\nu_{1}} \cdots \partial_{\nu_{s}} C_{\gamma_{1} \delta_{1} \cdots \gamma_{s} \delta_{s}}=0, \quad \partial_{\mu_{1}} \mathcal{B}^{\mu_{1} \cdots \mu_{s}}=0,
$$


where $\mathcal{B}^{\mu_{1} \cdots \mu_{s}}$ has dimension $s+2$ and is the totally symmetric traceless tensor in the $(s, 0, \ldots, 0)$ representation of $\mathrm{SO}(d)$ of dimension

$$
\mathrm{n}_{s}=(2 s+d-2) \frac{(s+d-3) !}{(d-2) ! s !},\left.\quad \mathrm{n}_{s}\right|_{d=4}=(s+1)^{2} .
$$

Thus, we need to subtract the term $(s+1)^{2} q^{s+2}$. We should also add back $s^{2} q^{s+3}$ to account for the conservation identity satisfied by $\mathcal{B}_{s}$, i.e. to compensate for the fact that the descendants of $\mathcal{B}_{s}$ containing dimension $s+3$ spin $s-1$ operator $\partial^{\mu_{1}} \mathcal{B}_{\mu_{1} \ldots \mu_{s}}$ are identically zero. In total, the generalization of the off-shell gauge-invariant operator count in (3.24) reads

$$
\mathcal{Z}_{s}^{\text {off-shell }}=\frac{2(2 s+1) q^{2}-(s+1)^{2} q^{s+2}+s^{2} q^{s+3}}{(1-q)^{4}} .
$$

The equations of motion for the conformal spin $s$ field following from (1.1) that generalize the linearized Bach equations (3.25) are

$$
B_{\mu_{1} \ldots \mu_{s}} \equiv \partial^{\nu_{1}} \ldots \partial^{\nu_{s}} C_{\mu_{1} \nu_{1} \ldots \mu_{s} \nu_{s}}=0, \quad \partial^{\mu_{1}} B_{\mu_{1} \ldots \mu_{s}}=0 .
$$

$B_{\mu_{1} \ldots \mu_{s}}$ has exactly the same properties as $\mathcal{B}^{\mu_{1} \cdots \mu_{s}}$ so its counting goes analogously, i.e. the generalization of (3.26) is found to be

$$
\mathcal{Z}_{s}^{\text {e.o.m. }}=\frac{(s+1)^{2} q^{s+2}-s^{2} q^{s+3}}{(1-q)^{4}} .
$$

Subtracting (4.16) from (4.14) as in (3.27), we get exactly the partition function in (4.8).

This provides strong support to our conjectures for the structure (4.1), (4.2) of the $s>2$ conformal higher spin operator on $S^{1} \times S^{3}$. An alternative derivation of $\mathcal{Z}_{s}$ based on group-theoretic argument will be presented in section 5 .

\section{Conformal spin $s$ partition function from $\mathrm{CFT}_{d} / \mathrm{AdS}_{d+1}$ perspective}

Let us now return to the discussion in the Introduction and elaborate on the relations (1.7), (1.15) between the conformal spin $s$ partition function and the partition functions associated with spin $s$ conformal operators in the singlet sector of free CFT in $d=4$ and thus also with the one-loop partition functions of massless spin $s$ gauge field in $\mathrm{AdS}_{5}$. We will suggest a method to compute the shadow partition function $\mathcal{Z}_{-s}$ that will allow a generalization to all even dimensions $d$ and will thus lead to the expression for the conformal higher spin partition function $\mathcal{Z}_{s}$ generalizing (4.8) to $d>4$.

\section{$5.1 d=4$ case}

The counting of the conformal higher spin equation of motion operators $B_{s}$ (4.15) discussed above is literally identical to the counting of spin $s$ conserved current operators $J_{s}$ in the singlet sector of free scalar $\mathrm{CFT}_{d}$ leading to the identification of $\mathcal{Z}_{s}^{\text {e.o.m. }}$ in (4.16) with $\mathcal{Z}_{+s}$ in (1.8). ${ }^{12}$ The latter should in turn be equal to the one-loop partition function

\footnotetext{
${ }^{12}$ The full large $N$ singlet-sector partition function in $d \geq 4$ is $\sum_{s=0}^{\infty} \mathcal{Z}_{+s}(q)=\left[\mathcal{Z}_{\text {c.s. }}(q)\right]^{2}$ which is the same as the one-loop partition function in the full higher spin theory in thermal AdS $_{d+1}$ (see [18, 33]).
} 
$\mathcal{Z}_{s}^{(+)}$of a massless spin $s$ field in thermal $\mathrm{AdS}_{d+1}$ with $S^{1} \times S^{d-1}$ boundary. Indeed, the expression $(1.10)$ or $(4.16)$ is the same as found in $[16,17]$ using the thermal quotient analog of massless spin $s$ field heat kernel in $\mathrm{AdS}_{5}$.

The direct computation of massless spin $s$ partition function with the alternative boundary conditions in $\operatorname{AdS}_{d+1}$ that should give $\mathcal{Z}_{s}^{(-)}$appears to be non-trivial. The final expression should match $\mathcal{Z}_{-s}$, i.e. the partition function that counts shadow operators of spin $s$ and dimension $\Delta_{-}=2-s$ in the scalar $\mathrm{CFT}_{d}$.

Below we shall first determine the expression for $\mathcal{Z}_{-s}$ by a somewhat heuristic approach and in the next section derive it by more rigorous group-theoretic method. The final $d=4$ expression will be the same as in (4.14) thus confirming the identification $\mathcal{Z}_{-s}=\mathcal{Z}_{s}^{\text {off }}$-shell between the shadow partition function and the off-shell part of the conformal higher spin partition function announced in (1.15).

As discussed in the Introduction, a naive guess (1.11) for $\mathcal{Z}_{-s}$ is obtained by replacing the dimensions in the expression (1.8) for $\mathcal{Z}_{+s}$ with their shadow counterparts, i.e. in $d=4$

$$
\widetilde{\mathcal{Z}}_{-s}(q)=\frac{(s+1)^{2} q^{2-s}-s^{2} q^{1-s}}{(1-q)^{4}}=\mathcal{Z}_{+s}(1 / q) .
$$

That may correspond to the analytic continuation in physical and ghost dimensions $(\Delta \rightarrow$ $d-\Delta)$ in the massless higher spin heat kernel expression for $\mathcal{Z}_{s}^{(+)}$on the $\mathrm{AdS}_{5}$ side. However, $\widetilde{\mathcal{Z}}_{-s}(q)$ containing poles in $q$ cannot be the final answer.

In general, the shadow field with dimension $\Delta_{-}=2-s$ corresponds to a conformal group $\mathrm{SO}(4,2)$ representation which is below the unitarity bound that may thus contain singular states and their associated submodules. From the $\mathrm{AdS}_{5}$ side, in the case of the alternative boundary conditions there are additional gauge transformations allowed by non-normalizability (see [14] and refs. there). These are in one-to-one correspondence with the conformal Killing tensors that belong to the $\mathrm{SO}(4,2)$ representation $(s-1, s-1,0)$ labelled by the Young tableau that has two rows with $s-1$ columns. ${ }^{13}$ Notice that this representation is finite dimensional and may be also described as a representation of the maximal compact real form $\mathrm{SO}(6)$, with the same Young tableau. The dimension of $(s-$ $1, s-1,0)$ is given in (4.10) with $d=6$ and $s \rightarrow s-1$, i.e.

$$
\left.\mathrm{n}_{(s-1, s-1,0)}\right|_{d=6}=\frac{1}{12} s^{2}(s+1)^{2}(2 s+1) .
$$

This suggests the following form for the full shadow partition function (justified on a grouptheoretic basis in appendix F.1)

$$
\mathcal{Z}_{-s}(q)=\widetilde{\mathcal{Z}}_{-s}(q)+\sigma_{s}(q)
$$

where $\sigma_{s}(q)$ is the character associated with the conformal algebra representation corresponding to the conformal Killing tensors. It may be computed as a specialization of the $\mathrm{SO}(6)$ character $\chi_{(s-1, s-1,0)}(\mathrm{x})$ (see appendix E), where we take a suitable limit of the

\footnotetext{
${ }^{13}$ Below we sometimes use also the simplified notation $(s-1, s-1) \equiv(s-1, s-1,0, \ldots, 0)$ for the 2 -row reprepresentation of $\mathrm{SO}(2 r)$.
} 
parameters $\mathrm{x}=\left(x_{1}, x_{2}, x_{3}\right)$ appropriate for counting the scaling dimensions of the states ${ }^{14}$ namely, $x_{1}=q$, and $x_{2}, x_{3} \rightarrow 1$ (see (F.24)). Explicitly, we have ${ }^{15}$

$$
\begin{aligned}
\sigma_{s}(q) & =\lim _{x \rightarrow 1} \chi_{(s-1, s-1,0)}(q, x, 1)=\lim _{x \rightarrow 1} \frac{\operatorname{det} M(s-1 ; x, q)}{\operatorname{det} M(0 ; x, q)}, \\
M(s-1 ; x, q) & =\left(\begin{array}{ccc}
2 & 2 & 1 \\
x^{s+1}+x^{-s-1} & x^{s}+x^{-s} & 1 \\
q^{s+1}+q^{-s-1} & q^{s}+q^{-s} & 1
\end{array}\right), \\
M(0 ; x, q) & =\left(\begin{array}{ccc}
2 & 2 & 1 \\
x^{2}+x^{-2} & x+x^{-1} & 1 \\
q^{2}+q^{-2} & q+q^{-1} & 1
\end{array}\right) .
\end{aligned}
$$

The ratio of the determinants in (5.4) can be expressed as

$$
\begin{aligned}
\sigma_{s}(q)= & \frac{1}{6} s(s+1)\left(s^{2}+s+1\right) \\
& -\frac{1}{6} \sum_{p=1}^{s-1} p(p+1)\left[(2 s+1) p-3 s^{2}-2 s-1\right]\left(q^{s-p}+q^{-s+p}\right) .
\end{aligned}
$$

Doing the finite sum and adding the result to (5.1), we finally obtain for $\mathcal{Z}_{-s}$ in (5.3)

$$
\mathcal{Z}_{-s}=\frac{2(2 s+1) q^{2}-(s+1)^{2} q^{s+2}+s^{2} q^{s+3}}{(1-q)^{4}}
$$

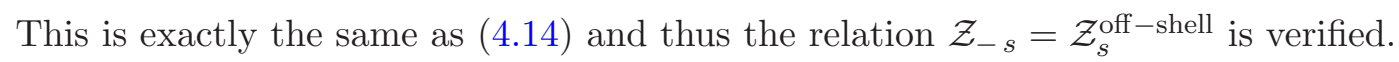

\subsection{Generalization to $d>4$}

The above computation of $\mathcal{Z}_{-s}$ can be readily generalized to any even $d>4$. We start with the known expression for $\mathcal{Z}_{+s}=\mathcal{Z}_{s}^{(+)}$in (1.8), i.e.

$$
\mathcal{Z}_{+s}=\frac{\mathrm{n}_{s} q^{s+d-2}-\mathrm{n}_{s-1} q^{s+d-1}}{(1-q)^{d}}
$$

that counts the conserved spin $s$ dimension $\Delta_{+}=s+d-2$ current operator and its descendants. The naive expression for the shadow partition function (1.11) is found by the $\Delta \rightarrow d-\Delta$ trick, resulting in $\widetilde{\mathcal{Z}}_{-s}(q)=\mathcal{Z}_{+s}(1 / q)$. The full $\mathcal{Z}_{-s}$ is obtained by correcting $\widetilde{\mathcal{Z}}_{-s}$ by the character of the conformal group $\mathrm{SO}(d, 2)$ representation associated with the conformal Killing tensors, i.e.

$$
\mathcal{Z}_{-s}(q)=\frac{\mathrm{n}_{s} q^{2-s}-\mathrm{n}_{s-1} q^{1-s}}{(1-q)^{d}}+\sigma_{s}(q), \quad \sigma_{s}(q)=\chi_{(s-1, s-1,0, \ldots, 0)}(q, 1, \ldots, 1) .
$$

\footnotetext{
${ }^{14}$ Here $\mathrm{x}$ may be associated with the Cartan generators of the compact subgroup $\mathrm{SO}(2) \times \mathrm{SO}(2) \times \mathrm{SO}(2) \subset$ $\mathrm{SO}(4,2)$, i.e., with the scaling dimension and two spins of $\mathrm{SO}(4)$.

${ }^{15}$ As recalled at the end of appendix $\mathrm{E}$, the character $\chi_{\ell}(\mathrm{x})$ is a combination of determinants that turns out to be a polynomial in the components $x_{i}$ of $\mathrm{x}$ and their inverses $x_{i}^{-1}$. So, the limit $x_{2}, x_{3} \rightarrow 1$ is smooth. Alternatively, working with (E.1), one has to set $x_{2}=x \rightarrow 1$ with $x_{3}=1$, as in (5.4).
} 
The corresponding $\mathfrak{s o}(d+2)$ character $\chi_{(s-1, s-1,0, \ldots, 0)}(q, 1, \ldots, 1)$ can be found from (E.1), (F.24), and the meaning of the choice of its arguments is like in the previous $d=4$ case (see also the discussion at the end of appendix E). As a result, we find that $\mathcal{Z}_{-s}(q)$ in (5.8) may be written in the following form with only positive powers of $q$ in the numerator

$$
\mathcal{Z}_{-s}(q)=\frac{1}{(1-q)^{d}}\left[\sum_{m=2}^{d-2}(-1)^{m} \mathrm{c}_{s, m} q^{m}-\mathrm{n}_{s} q^{s+d-2}+\mathrm{n}_{s-1} q^{s+d-1}\right] .
$$

We may thus represent $\mathcal{Z}_{-s}$ as

$$
\mathcal{Z}_{-s}(q)=\hat{\mathcal{Z}}_{s}(q)-\mathcal{Z}_{+s}(q), \quad \hat{\mathcal{Z}}_{s} \equiv \frac{1}{(1-q)^{d}} \sum_{m=2}^{d-2}(-1)^{m} \mathrm{c}_{s, m} q^{m} .
$$

Here the coefficients $\mathrm{n}_{s}$ are the same as in (1.9), (4.13), while $\mathrm{c}_{s, m}$ are some integers obeying $\mathrm{c}_{s, m}=\mathrm{c}_{s, d-m}$ that are readily found for any given value of $d$; their group theoretic interpretation as dimensions of $(s, s, 1, \ldots, 1,0, \ldots, 0)$ representations of $\mathrm{SO}(d)$ with $m-2$ rows of length 1 will be discussed in the next subsection. For example, in $d=4$ we have $\mathrm{n}_{s}=(s+1)^{2}$ and (cf. (5.6))

$$
\mathrm{c}_{s, 2}=2(2 s+1) .
$$

In $d=6$, where $\mathrm{n}_{s}=\frac{1}{12}(s+1)(s+2)^{2}(s+3)$ (see (4.13)), we find

$$
\mathrm{c}_{s, 2}=\mathrm{c}_{s, 4}=\frac{1}{12}(s+1)^{2}(s+2)^{2}(2 s+3), \quad \mathrm{c}_{s, 3}=\frac{1}{6} s(s+1)(s+2)(s+3)(2 s+3) .
$$

As a result, using (1.7), (5.7), (5.10), we arrive at the following expression for the conformal higher spin partition function in any even $d \geq 4$

$$
\begin{aligned}
\mathcal{Z}_{s}(q) & =\mathcal{Z}_{-s}(q)-\mathcal{Z}_{+s}(q)=\hat{\mathcal{Z}}_{s}(q)-2 \mathcal{Z}_{+s}(q) \\
& =\frac{1}{(1-q)^{d}}\left[\sum_{m=2}^{d-2}(-1)^{m} \mathrm{c}_{s, m} q^{m}-2 \mathrm{n}_{s} q^{s+d-2}+2 \mathrm{n}_{s-1} q^{s+d-1}\right] .
\end{aligned}
$$

This is a generalization of the $d=4$ expression in (1.12), (4.8).

The explicit form of the partition function (5.13) in general $d$ is readily found for few low values of $s$. The spin 0 case is special in that there are no gauge redundancies. Only the first terms in $\mathcal{Z}_{-s}(q)(5.9)$ and $\mathcal{Z}_{+s}(q)$ (1.8) are actually present, so the expression in (5.13) should be modified accordingly (the coefficient of the middle term in the bracket is 1 not 2 and the last term is absent). This gives

$$
\mathcal{Z}_{0}(q)=\frac{q^{2}-q^{d-2}}{(1-q)^{d}}
$$

For $s=1$ we get the following generalization of (2.4)

$$
\begin{aligned}
\mathcal{Z}_{+1} & =\frac{1}{(1-q)^{d}}\left(d q^{d-1}-q^{d}\right), \quad \mathcal{Z}_{-1}=\frac{1}{(1-q)^{d}} \sum_{m=2}^{d}(-1)^{m}\left(\begin{array}{c}
d \\
m
\end{array}\right) q^{m} \\
\mathcal{Z}_{1} & =\mathcal{Z}_{-1}-\mathcal{Z}_{+1}=\frac{1}{(1-q)^{d}}\left[\sum_{m=2}^{d-2}(-1)^{m}\left(\begin{array}{c}
d \\
m
\end{array}\right) q^{m}-2 d q^{d-1}+2 q^{d}\right] \\
& =1-\frac{1-d q-q^{d}+d q^{d-1}}{(1-q)^{d}}
\end{aligned}
$$


For $s=2$ we find

$$
\mathcal{Z}_{2}(q)=\frac{1}{2}\left[d^{2}+d+2+(d-2)(d+1) \frac{1-q^{d}}{(1-q)^{d}}\right]-d\left[\frac{q^{d}+(1-q)^{d}}{(1-q)^{d-1}}-\frac{1-(1-q)^{1-d}}{q}\right]
$$

These expressions can be checked by direct operator counting in the $d \geq 4$ conformal higher spin theory (1.2). For $s=0$ we have $C_{0}=\partial^{0} \phi_{0}=\phi_{0}$ of dimension 2 (see (1.3)) with the equations of motion being $\partial^{d-4} \phi_{0}=0$; this explains the two terms in the numerator of (5.14). $\mathcal{Z}_{0}$ in (5.14) vanishes in $d=4$ and is equal to the standard 2-derivative conformal scalar partition function $(2.3)$ in $d=6$.

For $s=1$ the first term in the sum in (5.15) is $\left(\begin{array}{l}d \\ 2\end{array}\right) q^{2}=\frac{1}{2} d(d-1) q^{2}$ which is the contribution of components of the dimension 2 field strength $C_{1}=\left(F_{\mu \nu}\right)$ of the conformal vector in $d$ dimensions with Lagrangian $F^{\mu \nu} \partial^{d-4} F_{\mu \nu}$, (see (1.2)). The second term $-\left(\begin{array}{l}d \\ 3\end{array}\right) q^{3}$ subtracts the contribution of the identity $\mathcal{B}^{\mu_{1} \ldots \mu_{d-3}}=\epsilon^{\mu_{1} \ldots \mu_{d}} \partial_{\mu_{d-2}} F_{\mu_{d-1} \mu_{d}}=0$ which has indeed $\left(\begin{array}{c}d \\ d-3\end{array}\right)=\left(\begin{array}{l}d \\ 3\end{array}\right)$ components. This overcounts as derivative of $\mathcal{B}^{\mu_{1} \ldots \mu_{d-3}}$ vanishes identically, so we need an extra terms to compensate for this, etc., etc. This explains the presence of all $d$ terms in the sum in $\mathcal{Z}_{-1}$ in (5.15). The first term in the numerator in $\mathcal{Z}_{+1}$ is the contribution of the equations of motion $B_{\mu}=\partial^{\nu} \partial^{d-4} F_{\mu \nu}=0$ where $B_{\mu}$ has $d$ components and dimension $d-1$. The second term accounts for the identity $\partial^{\mu} B_{\mu}=0$.

Similar counting can be repeated also for $s=2$ but is more cumbersome. These counting checks demonstrate consistency of the general expressions (5.8), (5.9), (5.13).

\subsection{Partition functions from characters of conformal algebra representations}

Our main result (5.13) for the conformal higher spin partition function can be understood at a deeper level in terms of characters of Verma modules of the conformal algebra $\mathfrak{s o}(d, 2)$ (see appendix $\mathrm{F}$ for details). From a group-theoretic perspective, the partition functions $\mathcal{Z}_{+s}$ and $\mathcal{Z}_{-s}$ are associated with the conformal current and the shadow fields. The important point is that the conformal current and the shadow field are not equivalent as $\mathfrak{s o}(d, 2)$ - modules. Instead, the conformal current generates a unitary irreducible module while the shadow field generates an indecomposable $\mathfrak{s o}(d, 2)$-module which is reducible (the Weyl-tensor like field strength built out of a shadow field is a conformal primary) and nonunitarizable (its dimension $\Delta_{-}=2-s$ is below the unitarity bound) [24]. The analysis of the relevant $\mathfrak{s o}(d, 2)$-modules has been presented in full generality in [20] where the corresponding resolutions à la Bernstein-Gelfand-Gelfand (BGG) have been derived. We have specialised it to our case in appendix $\mathrm{F}$, explaining in some detail various technical aspects.

The conformal spin $s$ partition function $\mathcal{Z}_{s}(q)=\mathcal{Z}_{-s}(q)-\mathcal{Z}_{+s}(q)$ can be interpreted as on-shell shadow field partition function, with $\mathcal{Z}_{-s}$ being the off-shell one. Eq. (F.21), i.e. $\mathcal{D}_{[2 ;(s, s)]}(q, \mathrm{x})=\mathcal{S}_{[2-s ;(s)]}(q, \mathrm{x})-\mathcal{D}_{[s+d-2 ;(s)]}(q, \mathrm{x})$, is relating the character corresponding to the dimension 2 conformal field in $(s, s)=(s, s, 0, \ldots, 0)$ representation of $\mathfrak{s o}(d)$ to the characters of the shadow field of dimension 2 and of the conserved current field of dimension $s+d-2$ both in $(s)=(s, 0, \ldots, 0)$ representations of $\mathfrak{s o}(d)$. The explicit expression for $\mathcal{D}_{[2 ;(s, s)]}(q, \mathrm{x})$ is given in eq. $(\mathrm{F} .23)$. 
To specify (F.23) to the case of the partition function counting only scaling dimensions (with no chemical potentials $x_{i}$ for $\mathfrak{s o}(d)$ spins) we need to set there $\mathrm{x}=(1, \ldots, 1)$. Then, according to (F.4), $P(q, \mathrm{x}) \rightarrow(1-q)^{-d}$. Also, in this case the $\mathfrak{s o}(d)$ characters $\chi_{\ell}(\mathrm{x})$ (see (E.1)) that appear in (F.23) read $\chi_{\ell}(1, \ldots, 1)$, i.e. are given by the dimension $\operatorname{dim}(\ell)$ of the corresponding finite-dimensional $\mathfrak{s o}(d)$ representation with Young tableau $\ell=\left(\ell_{1}, \ldots, \ell_{\frac{d}{2}}\right)$. The dimension of $(s)=(s, 0, \ldots, 0)$ representation of $\mathfrak{s o}(d)$ is given by $\mathrm{n}_{s}$ in (1.9), the dimension of $(s, s)=(s, s, 0, \ldots, 0)$ is given in (4.10), while the dimension of the representation $\left(s, s, 1^{p}\right)=(s, s, 1, \ldots, 1,0, \ldots, 0)$ associated with the Young tableau with two length $s$ rows and $p$ additional length 1 rows can be found from the algorithm in $[32]$.

Then, (F.23) takes exactly the same form as (5.9), with the coefficients $c_{s, m}$ in (5.9) now having an explicit group-theoretic interpretation as dimensions of the corresponding $\mathfrak{s o}(d)$ representations with two rows of length $s$ and $m-2$ rows of length 1 :

$$
\begin{aligned}
\mathrm{c}_{s, m} & =\operatorname{dim}\left(s, s, 1^{m-2}\right) \\
& =\frac{(2 s+d-2) !(s+d-3) !(s+d-4) !(s+d-3-m) !(s+m-3) !}{(2 s+d-5) !(s+m-1) !(s+d-1-m) ! s !(s-1) !(d-2) !(d-2-m) !(m-2) !} .
\end{aligned}
$$

Let us note also that the partition function corresponding to the conformal Killing tensors, given by $(\mathrm{F} .24)$ with $\mathrm{x}=(1, \ldots, 1)$, is the same as $\sigma_{s}(q)$ expressed in terms of the $\mathfrak{s o}(d+2)$ character in (5.8).

The above discussion applied to all even dimensions $d \geq 4$. Let us now comment on the special case of $d=2$. In $d=2$ the classical CHS action (1.2) is trivial (the $s>1$ field strengths vanish), so this partition function comes only from gauge freedom, i.e. from gauge fixing terms and ghosts in path integral approach (see also [10]). Assuming $s>1$, it follows from the general expression (5.13). For $d=2$ the first term in the bracket is absent and thus we get $\left(\mathrm{n}_{s}=2\right.$, see $\left.(4.13)\right)$

$$
\mathcal{Z}_{s}(q)=\frac{-4 q^{s}+4 q^{s+1}}{(1-q)^{2}}=-\frac{4 q^{s}}{1-q}, \quad s>1 .
$$

Equivalently, from (5.7), (5.8), (5.10),

$$
\mathcal{Z}_{s}=\mathcal{Z}_{-s}-\mathcal{Z}_{+s}=-2 \mathcal{Z}_{+s}, \quad \hat{\mathcal{Z}}_{s}=\mathcal{Z}_{-s}+\mathcal{Z}_{+s}=0, \quad s>1
$$

For $s=1$, i.e. the conformal vector field in $d=2$ with the non-local (Schwinger) action $\int d^{2} x F^{\mu \nu} \partial^{-2} F_{\mu \nu}$, the result is actually half of that of (5.18) with $s=1 .{ }^{16}$ If we also include dimension 2 scalar with the $\int d^{2} x \phi \partial^{-2} \phi$, then a similar count gives the numerator of $\mathcal{Z}_{0}$ as $q^{2}-1$, so that

$$
\mathcal{Z}_{1}=-\frac{2 q}{1-q}, \quad \mathcal{Z}_{0}=-\frac{1+q}{1-q}=\mathcal{Z}_{1}-1
$$

\footnotetext{
${ }^{16}$ Here the counting of terms in the numerator goes as follows: $F_{\mu \nu}$ of dimension 2 gives $q^{2}$, there are no gauge identities, while the equations of motion give $\partial^{-2} \partial^{\mu} F_{\mu \nu}=0, \partial^{-2} \partial^{\mu} \partial^{\nu} F_{\mu \nu}=0$, i.e. $2 q-q^{2}$. In total, $\mathcal{Z}_{1}=\frac{q^{2}-2 q+q^{2}}{(1-q)^{2}}=-\frac{2 q}{1-q}$.
} 
Equivalently, specializing the discussion of appendix $\mathrm{F}$ to the $\mathfrak{s o}(2,2)=\mathfrak{s o}(2,1) \oplus \mathfrak{s o}(2,1)$ case,one finds that the modules $\mathcal{V}_{[2 ;(s, s)]}$ and thus $\mathcal{D}_{[2 ;(s, s)]}$ are absent from the BGG sequence. The partition functions (characters) corresponding to the conserved current $[s ;(s)]$, shadow $[2-s ;(s)]$, conformal Killing tensor $[1-s ;(s-1)]$ and the conformal spin $s$ field $[2 ;(s, s)]$ representations of $\mathfrak{s o}(2,2)$ are then given, respectively, by

$$
\mathcal{Z}_{+s}=-2 C_{s-1}(q), \quad \mathcal{Z}_{-s}=2 C_{s-1}(q), \quad \sigma_{s}=2 \chi_{s-1}(q), \quad \mathcal{Z}_{s}=-4 C_{s-1}(q)
$$

where $C_{s}(q)=\frac{q^{s}}{1-q^{-1}}$ is the character of the $\mathfrak{s o}(3)$ Verma module corresponding to a highest weight $s$ representation. ${ }^{17}$

\section{Summing over spins: total CHS partion function and vanishing of Casimir energy}

Having found the conformal higher spin partition function for fixed $s$, i.e. (4.8) for $d=4$ and $(5.13)$ (with $(4.13),(5.17)$ ) for general $d$, we are ready to perform the sum over all spins $s=0,1,2, \ldots, \infty$ to find to the total CHS theory partition function.

The sum of the $\mathcal{Z}_{+s}$ part (1.8) of $\mathcal{Z}_{s}=\mathcal{Z}_{-s}-\mathcal{Z}_{+s}$ is finite (assuming $q<1$ )

$$
\mathcal{Z}_{+}(q)=\sum_{s=0}^{\infty} \mathcal{Z}_{+s}=\frac{q^{d-2}\left(1-q^{2}\right)^{2}}{(1-q)^{2 d}}
$$

Since $\mathcal{Z}_{+s}$ (5.7) is the same as the massless spin $s$ partition function in $A d S_{d+1}$, eq.(6.1) is equal to the partition function of totally symmetric massless higher spin Vasiliev theory. It is also the same as leading order term in the large $N$ limit of the canonical partition function of the singlet sector of $\mathrm{U}(N)$ scalar theory on $S^{1} \times S^{d-1}$ [18] (equivalently, it counts all spin $s$ conserved current operators in free scalar $\left.\mathrm{CFT}_{d}\right)$.

The sum of $\hat{\mathcal{Z}}_{s}$ in (5.10) and thus of $\mathcal{Z}_{-s}$ in (5.9) is, however, divergent: the coefficients $\mathrm{c}_{s, m}$ in $\hat{\mathcal{Z}}_{s}(5.10)$ are polynomials in $s$ (given by (5.17), (6.1)), and their growth with $s$ is not suppressed by $s$-independent powers of $q$. In the discussions of the conformal $a$-anomaly coefficient of CHS theory in $[9,10,14,15]$ it was noticed that there is a natural generalized $\zeta$-function or cutoff regularization [15] that leads to the vanishing of the total sum of the individual anomaly coefficients over $s:^{18}$

$$
\begin{array}{ccc}
\left.\sum_{s=0}^{\infty} e^{-\left(s+\frac{d-3}{2}\right) \epsilon} a_{s}\right|_{\epsilon \rightarrow 0, \text { fin }}=0, & \\
d=4: & a_{s}=\frac{1}{180} \nu^{2}(14 \nu+3), & \nu=s(s+1) ; \\
d=6: & a_{s}=\frac{1}{151200} \nu^{2}\left(22 \nu^{3}-55 \nu^{2}-2 \nu+2\right), & \nu=(s+1)(s+2) ;
\end{array}
$$

\footnotetext{
${ }^{17}$ Here $s$ may be any real number in general, see [19]. $\chi_{s}(q)$ is the standard $\mathfrak{s o}(3)$ character. Note also that $C_{s}\left(q^{-1}\right)=-C_{-(s+1)}(q), \quad C_{s-1}(q)+C_{s-1}\left(q^{-1}\right)=\chi_{s-1}(q)$.

${ }^{18}$ Here $\left.\right|_{\epsilon \rightarrow 0}$, fin means keeping only finite terms in the sum in the limit $\epsilon \rightarrow 0$, i.e. dropping all poles in $\epsilon$.
} 
This regularization should be consistent with the underlying symmetries of the CHS theory and so it is an obvious prescription to to define the total partition function. Then $\mathcal{Z}=$ $\left.\sum_{s=0}^{\infty} e^{-\left(s+\frac{d-3}{2}\right) \epsilon} \mathcal{Z}_{s}\right|_{\epsilon \rightarrow 0, \text { fin }}$ is given by

$$
\begin{aligned}
& \mathcal{Z}(q)=\hat{\mathcal{Z}}(q)-2 \mathcal{Z}_{+}(q) \\
& \hat{\mathcal{Z}}(q)=\frac{1}{(1-q)^{d}} \sum_{m=2}^{d-2}(-1)^{m} \hat{\mathrm{c}}_{m} q^{m},\left.\quad \hat{\mathrm{c}}_{m} \equiv \sum_{s=0}^{\infty} e^{-\left(s+\frac{d-3}{2}\right) \epsilon} \mathrm{c}_{s, m}\right|_{\epsilon \rightarrow 0, \text { fin }} .
\end{aligned}
$$

Using (5.11), (5.12), we thus find for $\mathcal{Z}=\sum_{s} \mathcal{Z}_{s}$

$$
\begin{aligned}
& d=4: \quad \mathcal{Z}=-\frac{q^{2}\left(11+26 q+11 q^{2}\right)}{6(1-q)^{6}} \\
& d=6: \quad \mathcal{Z}=\frac{q^{2}\left(407-5298 q-466311 q^{2}-992956 q^{3}-466311 q^{4}-5298 q^{5}+407 q^{6}\right)}{241920(1-q)^{10}} .
\end{aligned}
$$

In general, the summed expression has the same symmetry property as $\mathcal{Z}_{+}$in (6.1), i.e.

$$
\mathcal{Z}(q)=\mathcal{Z}(1 / q)
$$

Given the canonical partition function $\mathcal{Z}(q)(2.1)$, the corresponding Casimir energy on $S^{d-1}$ can be found from the standard relations (see, e.g., [34], cf. (2.1))

$$
E_{c}=\frac{1}{2} \sum_{n} \mathrm{~d}_{n} \omega_{n}=\frac{1}{2} \zeta_{E}(-1), \quad \zeta_{E}(z)=\frac{1}{\Gamma(z)} \int_{0}^{\infty} d \beta \beta^{z-1} \mathcal{Z}\left(e^{-\beta}\right) .
$$

Then, by the same argument as in the case of the massless higher spin partition function $\mathcal{Z}_{+}(q)$ in [18] it follows from (6.8) that the total summed over $s$ Casimir energy on $S^{d-1}$ in the conformal higher spin theory also vanishes.

It is of interest to derive this also directly from the explicit expressions for the Casimir energies of individual conformal spin fields. Let us start with the $d=4$ case. To compute the corresponding Casimir energy $E_{c, s}$ one may either use (6.9) with $\mathcal{Z}_{s}$ given by (4.8), or write $\mathcal{Z}_{s}$ as (noting that $\left.(1-q)^{-4}=\sum_{n=0}^{\infty}\left(\begin{array}{c}n+3 \\ 3\end{array}\right) q^{n}\right)$

$$
\mathcal{Z}_{s}=\sum_{n=0}^{\infty} \frac{1}{6}(n+1)(n+2)(n+3)\left[2(2 s+1) q^{n+2}-2(s+1)^{2} q^{n+s+2}+2 s^{2} q^{n+s+3}\right]
$$

and extract the corresponding degeneracies and mode energies to get $E_{c, s}=$ $\sum_{a} \sum_{n} \mathrm{~d}_{n}^{(a)} \omega_{n}^{(a)}$. Then, using the spectral cutoff or spectral $\zeta$-function regularization as standard for the Casimir energy (see also [18]), we find

$$
\begin{aligned}
E_{c, s}=\sum_{n=0}^{\infty} \frac{1}{12}(n+1)(n+2)(n+3)\left[2(2 s+1)(n+2) e^{-(n+2) \epsilon}\right. & \\
& \left.-2(s+1)^{2}(n+s+2) e^{-(n+s+2) \epsilon}+2 s^{2}(n+s+3) e^{-(n+s+3) \epsilon}\right]_{\epsilon \rightarrow 0, \text { fin }}
\end{aligned}
$$


so that

$$
d=4: \quad E_{c, s}=\frac{1}{720} \nu\left(18 \nu^{2}-14 \nu-11\right), \quad \nu=s(s+1) .
$$

Note that this expression is similar but different from (6.3) (in general, in $d>2$ the Casimir energy and the $a$-anomaly coefficients need not match, cf. the discussion in [18]). Still, the sum of $E_{c, s}$ also vanishes when computed with the same regularization as in (6.2):

$$
\left.\sum_{s=0}^{\infty} e^{-\left(s+\frac{d-3}{2}\right) \epsilon} E_{c, s}\right|_{\epsilon \rightarrow 0, \text { fin }}=0 .
$$

The same is true for any $d .{ }^{19}$ For example, in $d=6$ we find the following explicit expressions for $\mathcal{Z}_{s}$ in (5.13) and the corresponding Casimir energy (cf. (6.4))

$$
\begin{gathered}
\mathcal{Z}_{s}=\frac{(s+1)(s+2)}{12(1-q)^{6}}\left[(s+1)(s+2)(2 s+3) q^{2}-2 s(s+3)(2 s+3) q^{3}\right. \\
\left.+(s+1)(s+2)(2 s+3) q^{4}-2(s+2)(s+3) q^{s+4}+2 s(s+1) q^{s+5}\right], \\
d=6: \quad E_{c, s}=\frac{1}{241920} \nu^{2}\left(12 \nu^{3}-58 \nu^{2}-6 \nu+117\right), \quad \nu=(s+1)(s+2),
\end{gathered}
$$

and one can check directly that (6.13) is satisfied.

We remark that a different regularization of the sum over spins than used in (6.2) would have led to a partition function for which $\mathcal{Z}(q) \neq \mathcal{Z}(1 / q)$ and as a result the corresponding vacuum energy would have been non-vanishing. ${ }^{20}$ So, having total $E_{c}=0$ and having right regularization are correlated facts (see a related recent discussion in [35]).

Finally, let us consider the special case of $d=2(5.18),(5.19)$. In this case $\mathcal{Z}_{s}$ is $-2 \mathcal{Z}_{+s}$, i.e. is -2 of the massless spin $s$ partition function in $\mathrm{AdS}_{3}$, and we get from (5.18), (5.20) the following expression for the summed partition function

$$
d=2: \quad \mathcal{Z}=\mathcal{Z}_{0}+\mathcal{Z}_{1}+\sum_{s=2}^{\infty} \mathcal{Z}_{s}=-\frac{(1+q)^{2}}{(1-q)^{2}},
$$

so that once again $\mathcal{Z}(q)=\mathcal{Z}(1 / q)$ and the summed Casimir energy vanishes. This can be seen also explicitly using that $\mathcal{Z}_{s}=-2 \mathcal{Z}_{+s}$ implies that the corresponding Casimir energies are also proportional in the same way, i.e. (cf. eq. (5.31) in [18])

$$
d=2: \quad E_{c, s}=\frac{1}{6}[1+6 s(s-1)], \quad s>1 .
$$

In the special cases of "non-local" $s=0,1$ fields we get from (5.20) half the $s=0,1$ extrapolated values of (6.17), i.e. $E_{c, 0}=E_{c, 1}=\frac{1}{12}$. Using the universal regularisation (6.13) to define the sum over $s>1$, we conclude once again that the Casimir energy of the full CHS theory vanishes, ${ }^{21}$

$$
d=2: \quad E_{c, 0}+E_{c, 1}+\left.\sum_{s=2}^{\infty} e^{-\left(s-\frac{1}{2}\right) \epsilon} E_{c, s}\right|_{\epsilon \rightarrow 0, \text { fin }}=0 .
$$

\footnotetext{
${ }^{19}$ It is possible to check that the regularization with $e^{-(s+a) \epsilon}$ leads to vanishing sum over spins for any $d$ only if $a=\frac{d-3}{2}$.

${ }^{20}$ The regularization $(6.2),(6.13)$ is indeed a very natural one as one can show that it is a direct analog of the spectral regularization used in [15].

${ }^{21}$ This also follows from the fact that the total partition function is the function $\mathcal{Z}=\mathcal{Z}_{0}+\mathcal{Z}_{1}+\sum_{s=2}^{\infty} \mathcal{Z}_{s}=$ $-(1+q)^{2} /(1-q)^{2}$. Its symmetry under $q \rightarrow 1 / q$ explains the vanishing of the Casimir energy.
} 
In $d=2$ the Casimir energy on $S^{1}$ is indeed proportional to the corresponding conformal anomaly coefficient (central charge) which thus also cancels. ${ }^{22}$

\section{Concluding remarks}

In this paper we have found the partition function $\mathcal{Z}$ of non-interacting conformal higher spin (CHS) theory viewed as a collection of free spin $s$ CFT's in $\mathbb{R}^{d}$. The same partition function can be computed from the CHS theory defined on a curved conformally flat background $S^{1} \times S^{d-1}$. We discussed relations to partition functions appearing in the context of AdS/CFT and gave a representation-theoretic interpretation of $\mathcal{Z}$ in terms of characters of conformal algebra $\mathfrak{s o}(d, 2)$.

While we focused on the free CHS theory, we should stress that there exists a full nonlinear generalization. As is well known, introducing consistent interactions involving massless higher-spin fields is notoriously difficult and various no-go theorems express the incompatibility between higher-spin gauge symmetries and minimal coupling with gravity around a flat background (see, e.g., [36] for a review). Fradkin and Vasiliev discovered [37] that this incompatibility can be resolved on a constant curvature (A)dS background, and that eventually lead Vasiliev to his unfolded equations describing an interacting tower of massless higher-spin fields [38, 39]. Only two other explicit examples of interacting higherspin theories (in $d \geqslant 3$ ) are known at present: Chern-Simons-like theory in $d=3^{23}$ and conformal higher spin theory in even $d$ dimensions. In contradistinction with massless higher spin theory, these two share the following attractive features: their interactions are consistent with coupling to gravity even around a flat background and they admit a conventional action principle.

Indeed, the non-linear CHS theory can be defined as an induced theory [3, 4, 14, 42]. As mentioned in the Introduction, the logarithmically divergent piece of the one-loop effective action of a scalar CFT coupled to source fields $\phi_{s}$ for all conserved symmetric spin $s$ currents is a local functional of $\phi_{s}$ starting with the CHS kinetic term in (1.2) ${ }^{24}$ and can thus be interpreted as an action for the interacting CHS theory. ${ }^{25}$

As we have found above, the bosonic CHS theory has vanishing Casimir energy, and it may also be consistent at the quantum level if in addition to the vanishing of the anomaly $a$-coefficient $[9,14]$ the full conformal anomaly also vanishes. This theory may thus be worth a detailed exploration.

\footnotetext{
${ }^{22}$ The Seeley coefficient for a conformal $\operatorname{spin} s>1$ field is $B_{2}=\frac{1}{3} c=-\frac{2}{3}[1+6 s(s-1)]=-4 E_{c, s}$, see eq. (A.7) [10]. The same values follow [14] also from the $\mathrm{AdS}_{3}$ perspective, i.e. (1.6). For $s=1$ one finds $B_{2}=\frac{1}{3} c=-\frac{1}{3}[10]$. For the non-local scalar $\int \phi \partial^{-2} \phi$ the central charge is minus the value $(c=1)$ of the real scalar one, i.e. once again $B_{2}=-\frac{1}{3}$.

${ }^{23}$ The literature on this subject is considerable so we simply refer to $[40,41]$ for an example of CS theory in $d=3$ flat spacetime.

${ }^{24}$ More precisely, the vertex involving a product of $m$ fields with spins $s_{i}(i=1, \ldots, m)$ contains a total number $p=d+\sum_{i=1}^{m}\left(s_{i}-2\right)$ of partial derivatives [42]. As one can see, the spin-two fields do not contribute in the sum, consistently with minimal coupling to gravity (i.e. spin 2 fields essentially enter through general covariantization and do not modify the total number of derivatives).

${ }^{25}$ Notice that the resulting classical nonlinear theory is a metric-like one and does not require Vasiliev's unfolding procedure (though this alternative formulation should exist along the lines of [5]).
} 


\section{Acknowledgments}

XB thanks T. Basile, N. Boulanger, E. Joung for useful exchanges on group-theoretic issues and is especially grateful to O. Shaynkman for his patient introduction to [20]. AAT is grateful to S. Giombi, R. Metsaev and M. Vasiliev for discussions. The work of AAT was supported by the ERC Advanced grant No.290456 and also by the STFC grant $\mathrm{ST} / \mathrm{J} 000353 / 1$.

\section{A Spectrum of spin $s$ laplacian on $S^{d}$}

Let us summarize the result for the spectrum of the operator $\mathcal{O}_{s \perp}$ defined on symmetric traceless transverse tensors of rank $s$ on $S^{d}$

$$
\mathcal{O}_{s \perp}\left(M^{2}\right) \equiv\left(-D^{2}+M^{2}\right)_{s \perp}, \quad \mathcal{O}_{s \perp} \psi_{s}^{(n)}=\lambda_{n} \psi_{s}^{(n)} .
$$

The eigenvalues and their degeneracy are given by (see, e.g., $[43,44]$ )

$$
\begin{aligned}
\lambda_{n} & =(n+s)(n+s+d-1)-s+M^{2}, \quad n=0,1,2, \ldots, \\
\mathrm{d}_{n} & =g_{s} \frac{(n+1)(n+2 s+d-2)(2 n+2 s+d-1)(n+s+d-3) !}{(d-1) !(n+s+1) !} \\
g_{s} & =\frac{(2 s+d-3)(s+d-4) !}{(d-3) ! s !} .
\end{aligned}
$$

Here, $g_{s}$ is the number of components of the symmetric traceless transverse rank $s$ tensor in $d$ dimensions.

\section{B Expansions of curvature-squared invariants}

We used the following expressions [45] for the quadratic terms in the background field expansion of the curvature-squared terms appearing in the action of Weyl gravity

$$
\begin{aligned}
\delta^{2} \int d^{4} x \sqrt{g} R^{2}=\int d^{4} x \sqrt{g}[ & \frac{1}{2} R h_{\alpha \beta} D^{2} h^{\alpha \beta}+R R_{\alpha \mu \beta \nu} h^{\alpha \beta} h^{\mu \nu}-\frac{1}{4} R^{2} h_{\alpha \beta} h^{\alpha \beta} \\
& \left.+\left(R^{\alpha \beta} h_{\alpha \beta}\right)^{2}+R R^{\alpha}{ }_{\mu} h_{\alpha \beta} h^{\mu \beta}+\ldots\right], \\
\delta^{2} \int d^{4} x \sqrt{g} R_{\mu \nu} R^{\mu \nu}=\int d^{4} x \sqrt{g}\left[\frac{1}{4} h_{\alpha \beta} D^{2} h^{\alpha \beta}-\frac{1}{4} R_{\mu \nu} R^{\mu \nu} h_{\alpha \beta} h^{\alpha \beta}\right. & \text { B.1) } \\
& +R^{\alpha \mu \beta \nu} h_{\mu \nu} D^{2} h_{\alpha \beta}+\frac{1}{2} R^{\alpha \mu} R^{\beta \nu} h_{\alpha \beta} h_{\mu \nu} \\
& -\frac{1}{2} R^{\alpha \mu} R_{\mu \nu} h_{\alpha \beta} h^{\beta \nu}+R_{\mu \nu} R^{\rho \mu \beta \nu} h_{\alpha \rho} h_{\beta}^{\alpha} \\
& \left.+R^{\alpha \mu \beta \nu} R_{\alpha \rho \beta \tau} h_{\mu \nu} h^{\rho \tau}+\frac{1}{2} R^{\mu \nu} h_{\alpha \beta} D_{\mu} D_{\nu} h^{\alpha \beta}+\ldots\right],
\end{aligned}
$$

where dots stand for terms with covariant derivatives of $R_{\mu \nu}$ or vanishing due to the gauge conditions

$$
D^{\mu} h_{\mu \nu}=0, \quad h_{\mu}^{\mu}=0
$$


On conformally flat $d=4$ background we drop also terms containing the Weyl tensor

$$
C_{\alpha \beta \gamma \delta}=R_{\alpha \beta \gamma \delta}-\frac{1}{2}\left(g_{\alpha \gamma} R_{\delta \beta}-g_{\alpha \delta} R_{\gamma \beta}-g_{\beta \gamma} R_{\delta \alpha}+g_{\beta \delta} R_{\gamma \alpha}\right)-\frac{1}{6} R\left(g_{\alpha \delta} g_{\gamma \beta}-g_{\alpha \gamma} g_{\delta \beta}\right) .
$$

\section{Factorized form of Weyl graviton operator on an Einstein background}

For generality, let us also record the result (see also [46]) of expansion of the 2nd-derivative action (3.7) near a generic Einstein background $R_{\mu \nu}=\frac{1}{4} R g_{\mu \nu}$ which is not conformallyflat, i.e. may have a non-vanishing Weyl tensor (a simple example is $S^{2} \times S^{2}$ ). Then, the quadratic fluctuation action in (3.8) is given by

$$
\begin{aligned}
& \mathscr{L}_{\phi \phi}^{(2)}=-\frac{1}{4} \phi_{\mu \nu} \phi^{\mu \nu}, \quad \mathscr{L}_{h \phi}^{(2)}=\frac{1}{2} \phi^{\mu \nu} D^{2} h_{\mu \nu}-\frac{1}{12} R h^{\mu \nu} \phi_{\mu \nu}+R_{\mu \gamma \nu \delta} h^{\mu \nu} \phi^{\gamma \delta}, \\
& \mathscr{L}_{h h}^{(2)}=-\frac{1}{144} R^{2} h_{\mu \nu} h^{\mu \nu}+\frac{1}{12} R R_{\mu \gamma \nu \delta} h^{\mu \nu} h^{\gamma \delta}+\frac{1}{24} R h^{\mu \nu} D^{2} h_{\mu \nu} .
\end{aligned}
$$

Suppressing tensor indices, we get

$$
\begin{aligned}
\mathscr{L}^{(2)} & =\frac{1}{2} h \hat{\mathcal{O}} \phi-\frac{1}{4} \phi \phi+\frac{1}{24} R h \hat{\mathcal{O}} h, \\
\hat{\mathcal{O}} & =D^{2}+2 \mathscr{R}-\frac{1}{6} R, \quad h \mathscr{R} h \equiv h^{\mu \nu} R_{\mu \gamma \nu \delta} h^{\gamma \delta} .
\end{aligned}
$$

Integrating out $\phi$ we get $\mathscr{L}^{(2)}=\frac{1}{4} h \hat{\mathcal{O}}_{2} h$, where

$$
\hat{\mathcal{O}}_{2}=\left(\hat{\mathcal{O}}+\frac{1}{6} R\right) \hat{\mathcal{O}}=\left(-D^{2}-2 \mathscr{R}\right)\left(-D^{2}-2 \mathscr{R}+\frac{1}{6} R\right) .
$$

In particular, for the conformally-flat Einstein space like $S^{4}$ or $\mathrm{AdS}_{4}$ this reduces to the operator (3.4).

\section{On tensor zero modes on $S^{3}$}

To illustrate the reason for the truncation of the spectrum of $\mathcal{O}_{s}$ operators in the CHS partition function (4.5) on $S^{1} \times S^{3}$, let us consider explicitly the $s=2$ and $s=3$ cases.

In the $s=2$ case we may find the partition (3.16) in the $h_{0 \mu}=0, h_{\mu}^{\mu}=0$ gauge where we are left with the spatial traceless $h_{i j}$ tensor. We may split it as $h_{i j}=h_{i j}^{\mathrm{TT}}+D_{(i} V_{j)}$ where $h_{i j}^{\mathrm{TT}}$ is transverse traceless and $V_{i}$ is transverse, $D^{i} V_{i}=0$. Let us consider $V_{i}$ to be the $n$-th eigenfunction of the Laplacian on $S^{3}$, i.e. $\mathbf{D}^{2} V_{i}=-\lambda_{n} V_{i}$, where, according to $\left(\right.$ A.2), we have $\lambda_{n}=(n+1)(n+3)-1$. We want to show that for the mode $n=0$, we have $D_{(i} V_{j)}=0$, i.e. $V_{i}$ is a Killing vector on $S^{3}$, and therefore this mode does not appear in the decomposition of $h_{i j}$. Commuting the covariant derivatives on unit-radius $S^{3}$ one can show that

$$
\left(\mathbf{D}^{2}+\lambda_{n}-4\right) D_{(i} V_{j)}=0, \quad D^{i} D_{(i} V_{j)}=\left(2-\lambda_{n}\right) V_{j} .
$$

If $n=0$, then $\lambda_{n}=2$ and thus $D_{(i} V_{j)}$ is transverse. In this case, from (A.2) we see that $D_{(i} V_{j)}$ can be non-zero only if the equation $\left(n^{\prime}+2\right)\left(n^{\prime}+4\right)-2=-2$ admits a solution for some non-negative integer $n^{\prime}$. Since this is impossible, we have proved the above statement. 
The same mechanism works for spin 3 . Now, we split the symmetric traceless field $\phi_{i j k}$ in TT part plus traceless components as

$$
\begin{aligned}
\phi_{i j k} & =\phi_{i j k}^{\mathrm{TT}}+D_{(i} h_{j k)}^{\mathrm{TT}}+t_{i j k}, \\
t_{i j k} & =D_{(i} D_{j} V_{k)}-\frac{2}{5} g_{(i j} V_{k)}-\frac{1}{5} g_{(i j} \mathbf{D}^{2} V_{k)},
\end{aligned}
$$

where $V_{i}$ is again transverse. We want to show that certain low modes of $h_{i j}^{\mathrm{TT}}$ and $V_{i}$ drop from this decomposition. Let us begin with the first piece, $D_{(i} h_{j k)}^{\mathrm{TT}}$. Again, $h_{i j}^{\mathrm{TT}}$ can be assumed to be the $n$-th eigenfunction of the Laplacian on $S^{3}$, i.e. $\mathbf{D}^{2} h_{i j}^{\mathrm{TT}}=-\lambda_{n} h_{i j}^{\mathrm{TT}}$, where now $\lambda_{n}=(n+2)(n+4)-2$. As in (D.1) we get

$$
\left(\mathbf{D}^{2}+\lambda_{n}-6\right) D_{(k} h_{i j)}^{\mathrm{TT}}=0 .
$$

For the zero mode, $\lambda_{0}=6$ and $D_{(k} h_{i j)}^{\mathrm{TT}}=0$ follows from the positivity of the Laplacian and the fact that there are no constant 3 -tensors. Thus, this mode drops out from the splitting (D.2).

The analysis of the second term $t_{i j k}$ in (D.2) goes along similar lines. We assume $V_{i}$ to be the $n$-th mode of the Laplacian on $S^{3}, \mathbf{D}^{2} V_{i}=-\lambda_{n} V_{i}$, with $\lambda_{n}=(n+1)(n+3)-1$. We must drop the zero mode of $V_{i}$ as otherwise $t_{i j k}=0$, due to the analysis of the spin 2 case. To show that we must also drop the $n=1$ mode, we use the following relations, analogous to (D.1) and (D.3),

$$
\left(\mathbf{D}^{2}+\lambda_{n}-10\right) t_{i j k}=0, \quad D^{i} t_{i j k}=\frac{8}{5}\left(7-\lambda_{n}\right) D_{(j} V_{k)} .
$$

This shows that $t_{i j k}$ is transverse precisely at $n=1$ where $\lambda_{1}=7$. In this case, taking into account that the spectrum of $-\mathbf{D}^{2}$ on 3 -tensors is $\left(n^{\prime}+3\right)\left(n^{\prime}+5\right)-3$, we see that the operator $\mathbf{D}^{2}+\lambda_{1}-10 \equiv \mathbf{D}^{2}-3$ has no zero modes. Again, we conclude that $t_{i j k}=0$.

\section{E Characters of $\mathrm{SO}(d)$ representations}

Below we present the basic expression for the character of irreducible tensor representation of $\mathrm{SO}(d)$ with even $d=2 r$ (for details, see, e.g., [19]). The tensor representation is associated with a Young tableaux $\ell=\left(\ell_{1}, \ldots, \ell_{r}\right)$ with $r$ rows of length $\ell_{i}, i=1, \ldots, r$. The character $\chi_{\ell}(\mathrm{x})$ is the following symmetric function of $\mathrm{x}=\left(x_{1}, \ldots, x_{r}\right)$

$$
\chi_{\ell}(\mathrm{x})=\frac{\mathscr{D}_{+}(\mathrm{x})-\mathscr{D}_{-}(\mathrm{x})}{2 \mathscr{D}(\mathrm{x})}
$$

where

$$
\begin{aligned}
\mathscr{D}_{ \pm}(\mathrm{x}) & =\operatorname{det}\left(x_{i}^{k_{j}} \pm x_{i}^{-k_{j}}\right), \quad k_{j}=\ell_{j}+r-j \\
\mathscr{D}(\mathrm{x}) & =\prod_{1 \leq i<j \leq r}\left(x_{i}+x_{i}^{-1}-x_{j}-x_{j}^{-1}\right) .
\end{aligned}
$$

One can show that (E.1) leads to a polynomial in $x_{i}$ and $x_{i}^{-1}$ after an algebraic factorization. This follows from the general definition of the character as a trace. 
In the main text, we are interested in special cases where most of components $x_{i}$ are equal to 1 . This is a smooth unambiguous limit after factorization. If one starts directly with (E.1), one has to be careful to take a limit to avoid removable singularities, as we do in eq. (5.4).

\section{F Characters of relevant $\mathfrak{s o}(d, 2)$ representations}

Below we will explain the structure of spaces of some representations (i.e. modules) of the Lie algebra $\mathfrak{s o}(d, 2)$ of the conformal group in $d$ dimensions that are relevant for the computation of the CFT partition functions in the main text. By kinematics of vectorial $\mathrm{AdS}_{d+1} / \mathrm{CFT}_{d}$, these representations appear also in the description of massless symmetric higher spin $s \geqslant 1$ fields on $\mathrm{AdS}_{d+1}$. We will present the explicit expressions for the associated characters.

The structure of all (generalized) Verma modules ${ }^{26}$ of $\mathfrak{s o}(d, 2)$ has been described in [20]. The fundamental results of [20] are applied below to the particular $\mathfrak{s o}(d, 2)$-modules we are interested in here. ${ }^{27}$ While in the main text we consider only the case of even $d$, here we include for completeness also the case of odd $d$ which happens to be much simpler.

\section{F.1 Characters of generalized Verma $\mathfrak{s o}(d, 2)$-modules}

Let us first introduce some basic definitions and notation. The commutation relations of the generators of $\mathfrak{s o}(d, 2)$ can be cast in the form

$$
\begin{aligned}
{\left[\mathrm{E}, \mathrm{J}_{i}^{ \pm}\right] } & = \pm \mathrm{J}_{i}^{ \pm}, \quad\left[\mathrm{J}_{i j}, \mathrm{~J}_{k}^{ \pm}\right]=2 i \delta_{k[j} \mathrm{J}_{i]}^{ \pm} \\
{\left[\mathrm{J}_{i}^{-}, \mathrm{J}_{j}^{+}\right] } & =2\left(i \mathrm{~J}_{i j}+\delta_{i j} \mathrm{E}\right) \\
{\left[\mathrm{J}_{i j}, \mathrm{~J}_{k l}\right] } & =i \delta_{j k} \mathrm{~J}_{i l}+\text { antisymetrizations }
\end{aligned}
$$

where $i, j=1,2, \ldots, d$ and the ladder operators $\mathrm{J}_{i}^{ \pm}$shift the eigenvalues of $\mathrm{E}$ by \pm 1 .

The maximal compact subalgebra of $\mathfrak{s o}(d, 2)$ is the direct sum $\mathfrak{s o}(2) \oplus \mathfrak{s o}(d)=$ $\operatorname{span}\left\{\mathrm{E}, \mathrm{J}_{i j}\right\}$. The parabolic subalgebra $\operatorname{span}\left\{\mathrm{E}, \mathrm{J}_{i}^{-}, \mathrm{J}_{j k}\right\}$ is isomorphic to the algebra of homotheties of the Euclidean space $\mathbb{R}^{d}$. Consider the finite-dimensional irreducible module (to be denoted as $\mathcal{Y}_{[\Delta, \ell]}$ ) of the latter algebra which is characterized by the eigenvalue $\Delta$ of E, annihilated by $\mathrm{J}_{i}^{-}$and carrying a finite-dimensional representation of $\mathfrak{s o}(d)$ characterized by a weight vector $\ell \equiv\left(\ell_{1}, \ldots, \ell_{r-1}, \ell_{r}\right)$. Here $r$ denotes the rank of $\mathfrak{s o}(d)$ (i.e. the integer part of $d / 2$ ) and the labels satisfy $\ell_{1} \geqslant \ldots \geqslant \ell_{r-1} \geqslant\left|\ell_{r}\right| \geqslant 0$. When $d$ is even,

\footnotetext{
${ }^{26}$ Verma modules are highest-weight modules, i.e. they are generated by a highest weight vector. Strictly speaking, in the present paper we consider generalized Verma modules, i.e. freely generated by a (finite-dimensional) highest-weight space but we will drop the term "generalized" since this distinction is rather technical.

${ }^{27}$ More precisely, the general analysis presented in subsection 4.4 of [20] is particularized here to the modules in the first series associated with the dominant integral weight $\left(\lambda_{0}, \lambda_{1}, \ldots, \lambda_{d}\right)=(s-1, s-$ $1,0, \ldots, 0)$ in the notation of $[20]$.
} 
the last label $\ell_{r}$ can be positive or negative. Non-negative integer labels define a Young diagram where the length of the $i$-th row is $\ell_{i} .{ }^{28}$

The Verma $\mathfrak{s o}(d, 2)$-module $\mathcal{V}_{[\Delta, \ell]}$ is the module freely generated from the module $\mathcal{Y}_{[\Delta, \ell]}$ by the action of the raising operators $\mathrm{J}_{i}^{+}$. Following standard usage in the literature, we will often make use of the $\mathrm{CFT}_{d}$ language to describe the $\mathfrak{s o}(d, 2)$-modules although, strictly speaking, this language is adapted to the decomposition with respect to the noncompact subalgebra $\mathfrak{s o}(1,1) \oplus \mathfrak{s o}(d-1,1)$ in which case the raising and lowering operators are the translation and the conformal boost generators. Modulo this slight abuse of terminology, the Verma module $\mathcal{V}(\Delta, \ell)$ can be interpreted as the module spanned by the primary conformal field of scaling dimension $\Delta$ (or, equivalently, by $\mathcal{Y}_{[\Delta, \ell]}$ in the above notation) together with all its descendants. A descendant that is at the same time a primary is called a singular module.

The characters are generating functions of weight multiplicities via their power expansion in the variable $q$ corresponding to $\mathfrak{s o}(2)$ generated by $\mathrm{E}$ and of the variables $\mathrm{x}=\left(x_{1}, \ldots, x_{r}\right)$ generated by $\mathrm{J}_{i j}$. We shall denote the character by the same label as the corresponding module. The character of $\mathcal{Y}_{[\Delta, \ell]}$ can be written as

$$
\mathcal{Y}_{[\Delta, \ell]}(q, \mathrm{x})=q^{\Delta} \chi_{\ell}(\mathrm{x})
$$

where $\chi_{\ell}(x)$ is the usual character of the finite-dimensional $\mathfrak{s o}(d)$-module labelled by $\ell$ and recalled above in appendix E. The character of the Verma module $\mathcal{V}_{[\Delta, \ell]}$ is entirely determined by the character of $\mathcal{Y}_{[\Delta, \ell]}$ and by the character of the Verma module for the trivial weight $\mathcal{V}_{[0,0]}(q, \mathrm{x}) \equiv P(q, \mathrm{x})$, i.e.

$$
\mathcal{V}_{[\Delta, \ell]}(q, \mathrm{x})=\mathcal{Y}_{[\Delta, \ell]}(q, \mathrm{x}) \mathcal{V}_{[0,0]}(q, \mathrm{x})=q^{\Delta} \chi_{\ell}(\mathrm{x}) P(q, \mathrm{x})
$$

The explicit expression for $P(q, \mathrm{x})$ in even $d=2 r$ is (in odd $d=2 r+1$ there is an extra factor of $(1-q)^{-1}$, see, e.g., [19])

$$
P(q, \mathrm{x})=\prod_{i=1}^{r} \frac{1}{\left(1-q x_{i}\right)\left(1-q x_{i}^{-1}\right)} .
$$

\section{F.2 Odd dimension $d \geqslant 3$}

As usual, one may represent $\mathfrak{s o}(d, 2)$ as acting on fields in $\mathbb{R}^{d}$, i.e. interpret its representations in $\mathrm{CFT}_{d}$ language. The main representations considered below will be the conserved spin $s$ current one, the conformal spin $s$ or shadow field one and the conformal Killing tensor one.

Let us denote by $\mathcal{D}_{[1-s ;(s-1)]}$ the $\mathfrak{s o}(d, 2)$-module associated with the conformal Killing tensor fields in flat $d$-dimensional space which are totally symmetric tensors of rank $s-1$ and dimension $\Delta=1-s$ subject to the differential constraint: the traceless part of $\partial_{\left(i_{1}\right.} k_{\left.i_{2} \ldots i_{s}\right)}$

\footnotetext{
${ }^{28}$ In order to remove the exception of negative labels and deal, in the sequel, only with non-negative labels $\ell_{i} \geqslant 0(i=1, \ldots, r)$, we introduce the non-standard notation $\ell_{ \pm} \equiv\left(\ell_{1}, \ell_{2}, \ldots, \pm \ell_{r}\right)$ for the weights with nonvanishing last label when $d$ is even. With a slight abuse of notation, in this exceptional cases $\mathcal{Y}_{[\Delta, \ell]}$ will stand for the reducible module $\mathcal{Y}_{\left[\Delta, \ell_{+}\right]} \oplus \mathcal{Y}_{\left[\Delta, \ell_{-}\right]}$. The reducibility is then interpreted as the fact that a chirality condition can be imposed, e.g. (anti)selfduality on the $\ell_{\frac{d}{2}}$ columns of length $d / 2$.
} 
should vanish (cf. [47]). They may be interpreted as trivial gauge transformations of the conformal spin $s$ field $\phi_{s}$ in (1.2) or of the shadow field in $\mathrm{CFT}_{d}$ (and thus zero modes of the corresponding CHS ghost determinant). The corresponding finite-dimensional irreducible $\mathfrak{s o}(d, 2)$-module may be formally labelled also by the rectangular Young diagram $(s-1, s-1,0, \ldots, 0)$ of $\mathfrak{s o}(d+2)$ made of two rows of length $s-1$.

For odd dimension $d$, the resolution ${ }^{29}$ of $\mathcal{D}_{[1-s ;(s-1)]}$ à la Bernstein-Gelfand-Gelfand is the following exact sequence of $\mathfrak{s o}(d, 2)$-modules (cf. appendix B of [20]):

$$
\begin{aligned}
0 & \rightarrow \mathcal{V}_{[s+d-1 ;(s-1)]} \rightarrow \mathcal{V}_{[s+d-2 ;(s)]} \rightarrow \mathcal{V}_{[d-2 ;(s, s)]} \rightarrow \mathcal{V}_{[d-3 ;(s, s, 1)]} \\
& \rightarrow \mathcal{V}_{\left[d-4 ;\left(s, s, 1^{2}\right)\right]} \rightarrow \cdots \rightarrow \mathcal{V}_{\left[\frac{d+1}{2} ;\left(s, s, 1 \frac{d-5}{2}\right)\right]} \rightarrow \mathcal{V}_{\left[\frac{d-1}{2} ;\left(s, s, 1 \frac{d-5}{2}\right)\right]} \rightarrow \cdots \rightarrow \mathcal{V}_{\left[4 ;\left(s, s, 1^{2}\right)\right]} \\
& \rightarrow \mathcal{V}_{[3 ;(s, s, 1)]} \rightarrow \mathcal{V}_{[2 ;(s, s)]} \rightarrow \mathcal{V}_{[2-s ;(s)]} \rightarrow \mathcal{V}_{[1-s ;(s-1)]} \rightarrow \mathcal{D}_{[1-s ;(s-1)]} \rightarrow 0
\end{aligned}
$$

where $\left(s, s, 1^{p}\right)$ stands for a Young diagram with two rows of length $s$ and $p$ additional rows of length 1 . We also use shorthand notation $(s)=(s, 0, \ldots, 0)$ and $(s, s)=(s, s, 0, \ldots, 0)$.

Let us illustrate these homomorphisms by considering the first arrows in (F.5). The exactness implies that the map $\mathcal{V}_{[s+d-1 ;(s-1)]} \rightarrow \mathcal{V}_{[s+d-2 ;(s)]}$ is injective. In $\mathrm{CFT}_{d}$ language, the module $\mathcal{V}_{[s+d-2 ;(s)]}$ is generated by a traceless symmetric tensor $j^{\mu_{1} \ldots \mu_{s}}$ of conformal dimension $s+d-2$. Its divergence $\partial_{\nu} j^{\nu \mu_{1} \ldots \mu_{s-1}}$ is a primary field of conformal dimension $s+d-1$ and of rank $s-1$. The submodule generated by this primary field is indeed the image of $\mathcal{V}_{[s+d-1 ;(s-1)]}$ in $\mathcal{V}_{[s+d-2 ;(s)]}$. Moreover, the exactness also implies that this submodule is mapped by $\mathcal{V}_{[s+d-2 ;(s)]} \rightarrow \mathcal{V}_{[d-2 ;(s, s)]}$ to zero. The module $\mathcal{V}_{[d-2 ;(s, s)]}$ is generated by tensors $k^{\mu_{1} \ldots \mu_{s}, \nu_{1} \ldots \nu_{s}}$ of dimension $d-2$ with the symmetries of the spin- $s$ Weyl tensor. The $s$-th divergence $j^{\mu_{1} \ldots \mu_{s}} \equiv \partial_{\nu_{1}} \cdots \partial_{\nu_{s}} k^{\mu_{1} \ldots \mu_{s}, \nu_{1} \ldots \nu_{s}}$ is a primary field of dimension $s+d-2$ that can be interpreted as a trivial conserved current (an "improvement"). The submodule generated by this primary field is the image of $\mathcal{V}_{[s+d-2 ;(s)]}$ in $\mathcal{V}_{[d-2 ;(s, s)]}$, which is isomorphic to the quotient module $\mathcal{D}_{[s+d-2 ;(s)]}=\mathcal{V}_{[s+d-2 ;(s)]} / \mathcal{V}_{[s+d-1 ;(s-1)]}$ the representatives of which are conserved currents $j^{\mu_{1} \ldots \mu_{s}}$ since $\partial_{\nu} j^{\nu \mu_{1} \ldots \mu_{s-1}} \sim 0$ in $\mathcal{D}_{[s+d-2 ;(s)]}$. In other words, any strictly conserved current is trivial.

The maximal submodule of a reducible Verma $\mathfrak{s o}(d, 2)$-module arises from singular modules for $d$ odd and the resolution (F.5) of length $d$ provides the following recursive

\footnotetext{
${ }^{29}$ Let us recall few basic facts of homological algebra. A finite resolution of length $n \in \mathbb{N}$ of the module $V_{0}$ is an exact sequence of homomorphisms $d_{i}$

$$
0 \stackrel{d_{n+2}}{\rightarrow} V_{n+1} \stackrel{d_{n+1}}{\rightarrow} V_{n} \stackrel{d_{n}}{\rightarrow} \cdots \stackrel{d_{2}}{\rightarrow} V_{1} \stackrel{d_{1}}{\rightarrow} V_{0} \stackrel{d_{0}}{\rightarrow} 0
$$

A short exact sequence $0 \rightarrow V_{2} \rightarrow V_{1} \rightarrow V_{0} \rightarrow 0$ is a resolution of length 1 expressing that $V_{0}=V_{1} / V_{2}$. More generally, the exactness of the above sequence, i.e. Ker $d_{i}=\operatorname{Im} d_{i+1} \equiv D_{i}(i=0,1, \ldots, n+1)$, produces the following chain of short exact sequences

$$
0 \rightarrow D_{i+1} \rightarrow V_{i+1} \rightarrow D_{i} \rightarrow 0 \quad(i=0,1, \ldots, n)
$$

since $\operatorname{Im} d_{i}=V_{i} / \operatorname{Ker} d_{i}$. Notice that the first and last short exact sequence are degenerate and simply express that $D_{0}=V_{0}$ and $D_{n}=V_{n+1}$. The other members in this chain determine recursively all modules $D_{i}$ in terms of the $V_{j}$ with $j>i$. In particular, the module $V_{0}=D_{0}$ is resolved in the sense that $V_{0}=V_{1} /\left(V_{2} / \ldots\left(V_{n} / V_{n+1}\right)\right)$.
} 
chain of irreducible modules [20]:

$$
\begin{array}{rlrl}
\mathcal{D}_{[s+d-1 ;(s-1)]} & =\mathcal{V}_{[s+d-1 ;(s-1)]}, & \mathcal{D}_{[s+d-2 ;(s)]} & =\mathcal{V}_{[s+d-2 ;(s)]} / \mathcal{D}_{[s+d-1 ;(s-1)]}, \\
\mathcal{D}_{[d-2 ;(s, s)]} & =\mathcal{V}_{[2 ;(s, s)]} / \mathcal{D}_{[s+d-2 ;(s)]}, & \mathcal{D}_{[d-3 ;(s, s, 1)]} & =\mathcal{V}_{[3 ;(s, s, 1)]} / \mathcal{D}_{[2 ;(s, s)]}, \quad \ldots \\
\mathcal{D}_{[2 ;(s, s)]} & =\mathcal{V}_{[2 ;(s, s)]} / \mathcal{D}_{[3 ;(s, s, 1)]}, & \mathcal{D}_{[2-s ;(s)]} & =\mathcal{V}_{[2-s ;(s)]} / \mathcal{D}_{[2 ;(s, s)]}, \\
\mathcal{D}_{[1-s ;(s-1)]} & =\mathcal{V}_{[1-s ;(s-1)]} / \mathcal{D}_{[2-s ;(s)]}
\end{array}
$$

As we have seen, in $\mathrm{CFT}_{d}$ language the module $\mathcal{D}_{[s+d-2 ;(s)]}$ is spanned by a conserved current of spin $s$ together with all its descendants and the quotient by $\mathcal{D}_{[s+d-1 ;(s-1)]}$ in (F.6) is the translation of the conservation law. Similarly, $\mathcal{D}_{[2 ;(s, s)]}$ describes the module generated by the linearized Weyl-like tensor of a spin-s shadow field while $\mathcal{D}_{[3 ;(s, s, 1)]}$ in (F.8) corresponds to the generalized Bianchi identities. In turn, the module $\mathcal{D}_{[2-s ;(s)]}$ describes the module for a pure gauge shadow field since the quotient in (F.8) means that the Weyl curvature is set to zero. Finally, one recovers from (F.9) the initial identification of $\mathcal{D}_{[1-s ;(s-1)]}$ as the module of conformal Killing tensor fields, since the quotient by $\mathcal{D}_{[2-s ;(s)]}$ is the translation of the conformal Killing equation.

The chain (F.6)-(F.9) allows to compute the characters of all irreducible modules, e.g.,

$$
\begin{aligned}
& \mathcal{D}_{[s+d-2 ;(s)]}(q, \mathrm{x})=\mathcal{V}_{[s+d-2 ;(s)]}-\mathcal{V}_{[s+d-1 ;(s-1)]}(q, \mathrm{x}) \\
& =q^{s+d-2}\left(\chi_{(s)}(\mathrm{x})-q \chi_{(s-1)}(\mathrm{x})\right) P(q, \mathrm{x}), \\
& \mathcal{D}_{[1-s ;(s-1)]}(q, \mathrm{x})=\mathcal{V}_{[1-s ;(s-1)]}(q, \mathrm{x})+\mathcal{V}_{[s+d-1 ;(s-1)]}(q, \mathrm{x}) \\
& -\left(\mathcal{V}_{[2-s ;(s)]}(q, \mathrm{x})+\mathcal{V}_{[s+d-2 ;(s)]}(q, \mathrm{x})\right) \\
& +\sum_{p=0}^{\frac{d-5}{2}}(-1)^{p}\left(\mathcal{V}_{\left[2+p ;\left(s, s, 1^{p}\right)\right]}(q, \mathrm{x})+\mathcal{V}_{\left[d-2-p ;\left(s, s, 1^{p}\right)\right]}(q, \mathrm{x})\right) \\
& =\left[\left(q^{s+d-1}+q^{1-s}\right) \chi_{(s-1)}(\mathrm{x})-\left(q^{s+d-2}+q^{2-s}\right) \chi_{(s-1)}(\mathrm{x})\right. \\
& \left.+\sum_{p=0}^{\frac{d-5}{2}}(-1)^{p}\left(q^{2+p}+q^{d-2-p}\right) \chi_{\left(s, s, 1^{p}\right)}(\mathrm{x})\right] P(q, \mathrm{x}) .
\end{aligned}
$$

Let $\mathcal{S}_{[2-s ;(s)]}$ denote the $\mathfrak{s o}(d, 2)$-module generated by the shadow field of dimension $2-s$ and spin $s$. Notice that this module does not appear in the list (F.6)-(F.9), although it is irreducible for $d$ odd. ${ }^{30}$ Nevertheless, due to the previous identification of $\mathcal{D}_{[2-s ;(s)]}$ with the module of gauge transformations for a spin- $s$ shadow field, one finds that the character of the shadow field itself is given by

$$
\mathcal{S}_{[2-s ;(s)]}(q, \mathrm{x})=\mathcal{V}_{[2-s ;(s)]}(q, \mathrm{x})-\mathcal{D}_{[2-s ;(s)]}(q, \mathrm{x})
$$

\footnotetext{
${ }^{30}$ The natural description of shadow fields in this setting seems to be rather in terms of contragredient modules. In the computation of characters, one may ignore this subtlety.
} 


$$
\begin{aligned}
& =\mathcal{V}_{[2-s ;(s)]}(q, \mathrm{x})-\mathcal{V}_{[1-s ;(s-1)]}(q, \mathrm{x})+\mathcal{D}_{[1-s ;(s-1)]}(q, \mathrm{x}) \\
& =\mathcal{V}_{[s+d-1 ;(s-1)]}(q, \mathrm{x})-\mathcal{V}_{[s+d-2 ;(s)]}(q, \mathrm{x}) \\
& \quad+\sum_{p=0}^{\frac{d-5}{2}}(-1)^{p}\left[\mathcal{V}_{\left[2+p ;\left(s, s, 1^{p}\right)\right]}(q, \mathrm{x})+\mathcal{V}_{\left[d-2-p ;\left(s, s, 1^{p}\right)\right]}(q, \mathrm{x})\right],
\end{aligned}
$$

where (F.12) follows from the definition of the shadow field as a primary field modulo gauge symmetries, (F.13) comes from the second isomorphism in (F.9) and the value of the character (F.11) has been used to obtain the third equality (F.14).

\section{F.3 Even dimension $d \geqslant 4$}

For even dimension $d$, the situation is rather more intricate because the maximal submodule of a reducible Verma $\mathfrak{s o}(d, 2)$-module does not necessarily arises from singular modules only [20]. Moreover, the diagram of homomorphisms which is the analogue of (F.5) is not a mere line of arrows but a complicate diagram involving a rhombus in the middle and nonstandard arrows (cf. appendix B of [20]). For that reason, we will only provide the final result: the corresponding chain of irreducible modules. It has the same structure as (F.6)-(F.9) until its middle, but it differs in the lower half (cf. subsection 4.4.2 of [20])

$$
\begin{aligned}
& \mathcal{D}_{[s+d-1 ;(s-1)]}=\mathcal{V}_{[s+d-1 ;(s-1)]}, \quad \mathcal{D}_{[s+d-2 ;(s)]}=\mathcal{V}_{[s+d-2 ;(s)]} / \mathcal{D}_{[s+d-1 ;(s-1)]}, \quad \ldots \\
& \mathcal{D}_{\left[\frac{d}{2}+1 ;\left(s, s, 1^{\frac{d}{2}-3}\right)\right]}=\mathcal{V}_{\left[\frac{d}{2}+1 ;\left(s, s, 1^{\frac{d}{2}-3}\right)\right]} / \mathcal{D}_{\left[\frac{d}{2}+2 ;\left(s, s, 1^{\frac{d}{2}-4}\right)\right]}, \\
& \mathcal{D}_{\left[\frac{d}{2} ;\left(s, s, 1^{\frac{d}{2}-2}\right)_{ \pm}\right]}=\mathcal{V}_{\left[\frac{d}{2} ;\left(s, s, 1^{\frac{d}{2}-2}\right)_{ \pm}\right]} / \mathcal{D}_{\left[\frac{d}{2}+1 ;\left(s, s, 1^{\frac{d}{2}-3}\right)\right]}, \\
& \mathcal{D}_{\left[\frac{d}{2} ;\left(s, s, 1^{\frac{d}{2}-2}\right)\right]}=\mathcal{U}_{\left[\frac{d}{2} ;\left(s, s, 1^{\frac{d}{2}-2}\right)\right]} / \mathcal{V}_{\left[\frac{d}{2}+1 ;\left(s, s, 1^{\frac{d}{2}-3}\right)\right]}^{*}, \\
& \mathcal{D}_{\left[\frac{d}{2}-1 ;\left(s, s, 1 \frac{d}{2}-3\right)\right]}=\mathcal{V}_{\left[\frac{d}{2}-1 ;\left(s, s, 1 \frac{d}{2}-3\right)\right]} / \mathcal{U}_{\left[\frac{d}{2} ;\left(s, s, 12^{\frac{d}{2}-2}\right)\right]}=\mathcal{U}_{\left[\frac{d}{2}-1 ;\left(s, s, 1 \frac{d}{2}-3\right)\right]} / \mathcal{V}_{\left[\frac{d}{2}+2 ;\left(s, s, 1 \frac{d}{2}-4\right)\right]}^{*}, \cdots \\
& \mathcal{D}_{[2 ;(s, s)]}=\mathcal{V}_{[2 ;(s, s)]} / \mathcal{U}_{[3 ;(s, s, 1)]}=\mathcal{U}_{[2 ;(s, s)]} / \mathcal{V}_{[s+d-2 ;(s)]}^{*}, \\
& \mathcal{D}_{[2-s ;(s)]}=\mathcal{V}_{[2-s ;(s)]} / \mathcal{U}_{[2 ;(s, s)]}=\mathcal{U}_{[2-s ;(s)]} / \mathcal{V}_{[s+d-1 ;(s-1)]}^{*} \\
& \mathcal{D}_{[1-s ;(s-1)]}=\mathcal{V}_{[1-s ;(s-1)]} / \mathcal{U}_{[2-s ;(s)]},
\end{aligned}
$$

where $\mathcal{V}^{*}$ stands for the contragredient ${ }^{31}$ of the corresponding Verma module. The modules denoted by $\mathcal{U}$ correspond to reducible auxilliary modules.

In $\mathrm{CFT}_{d}$ language, the module $\mathcal{D}_{[s+d-2 ;(s)]}$ and $\mathcal{D}_{[1-s ;(s-1)]}$ keep their interpretations as conserved current and conformal Killing tensor. However, the pure gauge shadow field corresponds now to the auxilliary module $\mathcal{U}_{[2-s ;(s)]}$ in (F.18). The reason underlying this slight difference with $d$ odd case is that the module generated by a pure gauge field is reducible in even $d$ because there exist conformally-covariant gauge-fixing conditions. ${ }^{32}$

\footnotetext{
${ }^{31}$ The contragredient module is defined in subsection 4.3 of [20]. Roughly speaking, it is the dual space where the role of raising and lowering operators are interchanged. In CFT language, the contragredient analogue of a primary field is a constant field since it is annihilated by the translations.

${ }^{32}$ The simplest example is $s=1$ where the following descendant of a pure gauge field $A_{\mu}=\partial_{\mu} \varepsilon$ is also a primary field: $\square^{\frac{d}{2}-1} \partial \cdot A=\square^{\frac{d}{2}} \varepsilon$.
} 
Therefore, the irreducible module $\mathcal{D}_{[2-s ;(s)]}$ can be interpreted as a pure gauge shadow field obeying a suitable gauge-fixing condition corresponding to the quotient by $\mathcal{V}_{[d+s-1 ;(s-1)]}^{*}$ in (F.17). Similarly, in odd dimension $d$ the off-shell Weyl-like tensor of the spin- $s$ shadow field generates the irreducible module $\mathcal{D}_{[2 ;(s, s)]}$ but in even dimension $d$, the off-shell Weyllike tensor is reducible since conformally-covariant equations of motion (corresponding to the submodule $\mathcal{V}_{[s+d-2 ;(s)]}^{*}$ ) in (F.16) can be imposed. Indeed, in even dimension $d$ the irreducible module $\mathcal{D}_{[2 ;(s, s)]}$ correspond to the on-shell Weyl-like tensor for a shadow field of spin $s$.

Given the chain (F.15)-(F.18) of isomorphisms, one may again compute recusively the characters of all irreducible modules. The characters of the modules in the upper half of the chain are unchanged, e.g., (F.10) holds, but the characters in the lower half are slightly modified. For instance,

$$
\begin{aligned}
& \mathcal{D}_{[1-s ;(s-1)]}(q, \mathrm{x})=\mathcal{V}_{[1-s ;(s-1)]}(q, \mathrm{x})+\mathcal{V}_{[s+d-1 ;(s-1)]}(q, \mathrm{x}) \\
& -\left(\mathcal{V}_{[2-s ;(s)]}(q, \mathrm{x})+\mathcal{V}_{[s+d-2 ;(s)]}(q, \mathrm{x})\right) \\
& +\sum_{p=0}^{\frac{d}{2}-3}(-1)^{p}\left(\mathcal{V}_{\left[2+p ;\left(s, s, 1^{p}\right)\right]}(q, \mathrm{x})+\mathcal{V}_{\left[d-2-p ;\left(s, s, 1^{p}\right)\right]}(q, \mathrm{x})\right) \\
& +(-1)^{\frac{d}{2}} \mathcal{V}_{\left[\frac{d}{2} ;\left(s, s, 1^{\frac{d}{2}-2}\right)\right]}(q, \mathrm{x}) \\
& =\left[\left(q^{s+d-1}+q^{1-s}\right) \chi_{(s-1)}(\mathrm{x})-\left(q^{s+d-2}+q^{2-s}\right) \chi_{(s-1)}(\mathrm{x})\right. \\
& \left.+\sum_{p=0}^{\frac{d}{2}-3}(-1)^{p}\left(q^{2+p}+q^{d-2-p}\right) \chi_{\left(s, s, 1^{p}\right)}(\mathrm{x})+(-q)^{\frac{d}{2}} \chi_{\left(s, s, 1^{\frac{d}{2}-2}\right)}(\mathrm{x})\right] P(q, \mathrm{x})
\end{aligned}
$$

Due to the identification of $\mathcal{U}_{[2-s ;(s)]}$ with the module of gauge transformations for a spin- $s$ shadow field, one finds that the character of the module $\mathcal{S}_{[2-s ;(s)]}$ generated by the shadow field itself is given by

$$
\mathcal{S}_{[2-s ;(s)]}(q, \mathrm{x})=\mathcal{V}_{[2-s ;(s)]}(q, \mathrm{x})-\mathcal{U}_{[2-s ;(s)]}(q, \mathrm{x}) .
$$

Notice that the important relation (F.13) for $\mathcal{S}_{[2-s ;(s)]}(q, \mathrm{x})$ (the counterpart of the prescription for $\mathcal{Z}_{-s}$ proposed in the introduction) holds also for $d$ even, as can be seen from (F.20) and (F.18). The explicit expression for this character can be easily computed from (F.3) and (F.19). By making use of the isomorphisms (F.16)-(F.18) together with the equalities (F.10) and (F.20), one can prove the following remarkable identity (the counterpart of the relation in (1.7))

$$
\mathcal{D}_{[2 ;(s, s)]}(q, \mathrm{x})=\mathcal{S}_{[2-s ;(s)]}(q, \mathrm{x})-\mathcal{D}_{[s+d-2 ;(s)]}(q, \mathrm{x}) .
$$

It is consistent with the expected isomorphism

$$
\mathcal{D}_{[2 ;(s, s)]}=\mathcal{S}_{[2-s ;(s)]} / \mathcal{D}_{[s+d-2 ;(s)]},
$$


where the quotient by $\mathcal{D}_{[s+d-2 ;(s)]}$ is the translation of the imposition of the equations of motion of order $d+2 s-4$ on the spin- $s$ shadow field, or, equivalently, of the conformal higher spin equations corresponding to $(1.2)[1,4]$ (see also [5, 24]). Another way to understand the appearance of $\mathcal{D}_{[s+d-2 ;(s)]}$ is that the natural source term for such equations is indeed a conformal current that we set to zero when we impose them.

The equations (F.19), (F.20) and (F.21) allow us to find the important character of the on-shell conformal field:

$$
\begin{aligned}
\mathcal{D}_{[2 ;(s, s)]}(q, \mathrm{x})= & 2\left(\mathcal{V}_{[s+d-1 ;(s-1)]}(q, \mathrm{x})-\mathcal{V}_{[s+d-2 ;(s)]}(q, \mathrm{x})\right)+(-1)^{\frac{d}{2}} \mathcal{V}_{\left[\frac{d}{2} ;\left(s, s, 1^{\frac{d}{2}-2}\right)\right]}(q, \mathrm{x}) \\
& +\sum_{p=0}^{\frac{d}{2}-3}(-1)^{p}\left(\mathcal{V}_{\left[2+p ;\left(s, s, 1^{p}\right)\right]}(q, \mathrm{x})+\mathcal{V}_{\left[d-2-p ;\left(s, s, 1^{p}\right)\right]}(q, \mathrm{x})\right) \\
= & {\left[2 q^{s+d-2}\left(q \chi_{(s-1)}(\mathrm{x})-\chi_{(s-1)}(\mathrm{x})\right)\right.} \\
& \left.+\sum_{p=0}^{\frac{d}{2}-3}(-1)^{p}\left(q^{2+p}+q^{d-2-p}\right) \chi_{\left(s, s, 1^{p}\right)}(\mathrm{x})+(-q)^{\frac{d}{2}} \chi_{\left(s, s, 1^{\frac{d}{2}-2}\right)}(\mathrm{x})\right] P(q, \mathrm{x}) .
\end{aligned}
$$

Finally, let us note also that there is the following relation between the relevant characters of $\mathfrak{s o}(d, 2)$ and $\mathfrak{s o}(d+2)$ for the finite-dimensional representations

$$
\mathcal{D}_{[1-s ;(s-1)]}\left(q, x_{1}, \ldots, x_{\frac{d}{2}}\right)=\chi_{(s-1, s-1)}\left(q, x_{1}, \ldots, x_{\frac{d}{2}}\right),
$$

where $\chi_{(s-1, s-1)}\left(y_{1}, y_{2}, \ldots, y_{\frac{d}{2}}, y_{\frac{d+2}{2}}\right)$ is the character of 2-row $(s-1, s-1) \equiv(s-1, s-$ $1,0, \ldots, 0)$ representation of $\mathfrak{s o}\left(d^{2}+2\right)$ defined in appendix E.

Open Access. This article is distributed under the terms of the Creative Commons Attribution License (CC-BY 4.0), which permits any use, distribution and reproduction in any medium, provided the original author(s) and source are credited.

\section{References}

[1] E.S. Fradkin and A.A. Tseytlin, Conformal supergravity, Phys. Rept. 119 (1985) 233 [INSPIRE].

[2] E.S. Fradkin and V.Y. Linetsky, Cubic interaction in conformal theory of integer higher spin fields in four-dimensional space-time, Phys. Lett. B 231 (1989) 97 [INSPIRE].

[3] A.A. Tseytlin, On limits of superstring in $A d S_{5} \times S^{5}$, Theor. Math. Phys. 133 (2002) 1376 [hep-th/0201112] [INSPIRE].

[4] A.Y. Segal, Conformal higher spin theory, Nucl. Phys. B 664 (2003) 59 [hep-th/0207212] [INSPIRE].

[5] M.A. Vasiliev, Bosonic conformal higher-spin fields of any symmetry, Nucl. Phys. B 829 (2010) 176 [arXiv:0909.5226] [INSPIRE].

[6] R. Marnelius, Lagrangian conformal higher spin theory, arXiv:0805.4686 [INSPIRE]. 
[7] J.L. Cardy, Operator content and modular properties of higher dimensional conformal field theories, Nucl. Phys. B 366 (1991) 403 [inSPIRE].

[8] D. Kutasov and F. Larsen, Partition sums and entropy bounds in weakly coupled CFT, JHEP 01 (2001) 001 [hep-th/0009244] [INSPIRE].

[9] A.A. Tseytlin, On partition function and Weyl anomaly of conformal higher spin fields, Nucl. Phys. B 877 (2013) 598 [arXiv:1309.0785] [INSPIRE].

[10] A.A. Tseytlin, Weyl anomaly of conformal higher spins on six-sphere, Nucl. Phys. B 877 (2013) 632 [arXiv:1310.1795] [INSPIRE].

[11] R.R. Metsaev, Arbitrary spin conformal fields in (A)dS, arXiv:1404.3712 [INSPIRE].

[12] T. Nutma and M. Taronna, On conformal higher spin wave operators, JHEP 06 (2014) 066 [arXiv: 1404.7452] [INSPIRE].

[13] H. Liu and A.A. Tseytlin, $D=4$ super Yang-Mills, $D=5$ gauged supergravity and $D=4$ conformal supergravity, Nucl. Phys. B 533 (1998) 88 [hep-th/9804083] [INSPIRE].

[14] S. Giombi, I.R. Klebanov, S.S. Pufu, B.R. Safdi and G. Tarnopolsky, AdS description of induced higher-spin gauge theory, JHEP 10 (2013) 016 [arXiv:1306.5242] [INSPIRE].

[15] S. Giombi, I.R. Klebanov and B.R. Safdi, Higher spin $A d S_{d+1} / C F T_{d}$ at one loop, Phys. Rev. D 89 (2014) 084004 [arXiv:1401.0825] [INSPIRE].

[16] R. Gopakumar, R.K. Gupta and S. Lal, The heat kernel on AdS, JHEP 11 (2011) 010 [arXiv: 1103.3627] [INSPIRE].

[17] R.K. Gupta and S. Lal, Partition functions for higher-spin theories in AdS, JHEP 07 (2012) 071 [arXiv: 1205.1130] [INSPIRE].

[18] S. Giombi, I.R. Klebanov and A.A. Tseytlin, Partition functions and Casimir energies in higher spin $A d S_{d+1} / C F T_{d}$, Phys. Rev. D 90 (2014) 024048 [arXiv:1402.5396] [INSPIRE].

[19] F.A. Dolan, Character formulae and partition functions in higher dimensional conformal field theory, J. Math. Phys. 47 (2006) 062303 [hep-th/0508031] [INSPIRE].

[20] O.V. Shaynkman, I.Y. Tipunin and M.A. Vasiliev, Unfolded form of conformal equations in $M$ dimensions and $o(M+2)$ modules, Rev. Math. Phys. 18 (2006) 823 [hep-th/0401086] [INSPIRE].

[21] R.R. Metsaev, Shadows, currents and AdS, Phys. Rev. D 78 (2008) 106010 [arXiv: 0805.3472] [INSPIRE].

[22] R.R. Metsaev, Gauge invariant two-point vertices of shadow fields, AdS/CFT and conformal fields, Phys. Rev. D 81 (2010) 106002 [arXiv:0907.4678] [INSPIRE].

[23] R.R. Metsaev, Anomalous conformal currents, shadow fields and massive AdS fields, Phys. Rev. D 85 (2012) 126011 [arXiv:1110.3749] [INSPIRE].

[24] X. Bekaert and M. Grigoriev, Notes on the ambient approach to boundary values of AdS gauge fields, J. Phys. A 46 (2013) 214008 [arXiv:1207.3439] [INSPIRE].

[25] A.A. Tseytlin, Effective action in De Sitter space and conformal supergravity, Yad. Fiz. 39 (1984) 1606 [Sov. J. Nucl. Phys. 39 (1984) 1018].

[26] E.S. Fradkin and A.A. Tseytlin, Instanton zero modes and $\beta$-functions in supergravities. 2. Conformal supergravity, Phys. Lett. B 134 (1984) 307 [INSPIRE]. 
[27] S. Deser and R.I. Nepomechie, Gauge invariance versus masslessness in de Sitter space, Annals Phys. 154 (1984) 396 [INSPIRE].

[28] S. Deser and R.I. Nepomechie, Anomalous propagation of gauge fields in conformally flat spaces, Phys. Lett. B 132 (1983) 321 [InSPIRE].

[29] R.R. Metsaev, Ordinary-derivative formulation of conformal low spin fields, JHEP 01 (2012) 064 [arXiv:0707.4437] [INSPIRE].

[30] R.R. Metsaev, Ordinary-derivative formulation of conformal totally symmetric arbitrary spin bosonic fields, JHEP 06 (2012) 062 [arXiv:0709.4392] [INSPIRE].

[31] M. Kaku, P.K. Townsend and P. van Nieuwenhuizen, Gauge theory of the conformal and superconformal group, Phys. Lett. B 69 (1977) 304 [INSPIRE].

[32] R.C. King and N.E. Samra, Dimensions of irreducible representations of the classical Lie groups, J. Phys. A 12 (1979) 2317.

[33] S.H. Shenker and X. Yin, Vector models in the singlet sector at finite temperature, arXiv: 1109.3519 [INSPIRE].

[34] G.W. Gibbons, M.J. Perry and C.N. Pope, Partition functions, the Bekenstein bound and temperature inversion in Anti-de Sitter space and its conformal boundary,

Phys. Rev. D 74 (2006) 084009 [hep-th/0606186] [INSPIRE].

[35] G. Basar, A. Cherman, D.A. McGady and M. Yamazaki, T-reflection, arXiv:1406.6329 [INSPIRE].

[36] X. Bekaert, N. Boulanger and P. Sundell, How higher-spin gravity surpasses the spin two barrier: no-go theorems versus yes-go examples, Rev. Mod. Phys. 84 (2012) 987 [arXiv: 1007.0435] [INSPIRE].

[37] E.S. Fradkin and M.A. Vasiliev, On the gravitational interaction of massless higher spin fields, Phys. Lett. B 189 (1987) 89 [INSPIRE].

[38] M.A. Vasiliev, Consistent equation for interacting gauge fields of all spins in (3+1)-dimensions, Phys. Lett. B 243 (1990) 378 [InSPIRE].

[39] M.A. Vasiliev, Nonlinear equations for symmetric massless higher spin fields in (A)dS(d), Phys. Lett. B 567 (2003) 139 [hep-th/0304049] [INSPIRE].

[40] H. Afshar, A. Bagchi, R. Fareghbal, D. Grumiller and J. Rosseel, Spin-3 gravity in three-dimensional flat space, Phys. Rev. Lett. 111 (2013) 121603 [arXiv: 1307.4768] [INSPIRE].

[41] H.A. Gonzalez, J. Matulich, M. Pino and R. Troncoso, Asymptotically flat spacetimes in three-dimensional higher spin gravity, JHEP 09 (2013) 016 arXiv:1307.5651] [INSPIRE].

[42] X. Bekaert, E. Joung and J. Mourad, Effective action in a higher-spin background, JHEP 02 (2011) 048 [arXiv: 1012.2103] [INSPIRE].

[43] A. Higuchi, Symmetric tensor spherical harmonics on the $N$ sphere and their application to the de Sitter group $\mathrm{SO}(N, 1)$, J. Math. Phys. 28 (1987) 1553 [Erratum ibid. 43 (2002) 6385] [INSPIRE].

[44] R. Camporesi and A. Higuchi, Spectral functions and zeta functions in hyperbolic spaces, J. Math. Phys. 35 (1994) 4217 [INSPIRE].

[45] N.H. Barth and S.M. Christensen, Quantizing fourth order gravity theories. 1. The functional integral, Phys. Rev. D 28 (1983) 1876 [INSPIRE]. 
[46] S. Deser, E. Joung and A. Waldron, Partial masslessness and conformal gravity, J. Phys. A 46 (2013) 214019 [arXiv:1208.1307] [inSPIRE].

[47] M.G. Eastwood, Higher symmetries of the Laplacian, Annals Math. 161 (2005) 1645 [hep-th/0206233] [INSPIRE]. 